\title{
Particle Spectra from Acceleration at Forward and Reverse Shocks of Young Type Ia Supernova Remnants
}

\author{
I.Telezhinsky ${ }^{\mathrm{a}}$, V.Dwarkadas ${ }^{\mathrm{b}}$, M.Pohl $^{\mathrm{a}, \mathrm{c}}$ \\ ${ }^{a}$ DESY, Platanenallee 6, 15738 Zeuthen, Germany \\ ${ }^{b}$ University of Chicago, Department of Astronomy \& Astrophysics, 5640 S Ellis Ave, AAC 010c, Chicago, \\ IL 6063\%, U.S.A. \\ ${ }^{c}$ Universität Potsdam, Institut für Physik 8 Astronomie, Karl-Liebknecht-Strasse 24/25, 14476 Potsdam, \\ Germany
}

\begin{abstract}
We study cosmic-ray acceleration in young Type Ia Supernova Remnants (SNRs) by means of test-particle diffusive shock acceleration theory and 1-D hydrodynamical simulations of their evolution. In addition to acceleration at the forward shock, we explore the particle acceleration at the reverse shock in the presence of a possible substantial magnetic field, and consequently the impact of this acceleration on the particle spectra in the remnant. We investigate the time evolution of the spectra for various time-dependent profiles of the magnetic field in the shocked region of the remnant. We test a possible influence on particle spectra of the Alfvénic drift of scattering centers in the precursor regions of the shocks. In addition, we study the radiation spectra and morphology in a broad band from radio to gamma-rays. It is demonstrated that the reverse shock contribution to the cosmic-ray particle population of young Type Ia SNRs may be significant, modifying the spatial distribution of particles and noticeably affecting the volume-integrated particle spectra in young SNRs. In particular spectral structures may arise in test-particle calculations that are often discussed as signatures of non-linear cosmic-ray modification of shocks. Therefore, the spectrum and morphology of emission, and their time evolution, differ from pure forward-shock solutions.
\end{abstract}

Keywords: Supernova Remnants; cosmic rays; cosmic-ray acceleration; hydrodynamics; forward and reverse shocks

\section{Introduction}

The energy density in local cosmic rays (CRs), when extrapolated to the whole Galaxy, implies the existence of powerful accelerators inside the Galaxy. Supernova Remnants (SNRs) have long been thought to be the main candidates on account of energetics [1]. Later, diffusive shock acPreprint submitted to Astroparticle Physics celeration (DSA) of charged particles at the SNR shocks was considered [2, 3, 4, , 5] and is now thought to be the most likely mechanism that can accelerate CRs up to $10^{15} \mathrm{eV}$, where the cosmic-ray spectrum shows a break known as the 'knee' [6]. If they are very efficiently accelerated, CRs can produce a feedback on the plasma flow 
in SNRs through their pressure, and can modify the shock structure by decelerating the incoming plasma flow when streaming through it in the precursor region (see [7] for a review and references therein). Observationally, it is unclear whether or not the CR-acceleration efficiency is sufficiently high for a significant feedback [8, 9], but if so, SNR shocks may become modified, and the particle spectra may deviate from the classical power-law slope of $s=2$ found in test-particle calculations. The spectra produced by modified shocks show concave structure with softer $(s>2)$ low-energy and harder $(s<2)$ high-energy parts.

The CR spectrum observed at Earth suggests that sources produce softer spectra than predicted by non-linear DSA (NDSA) theory [10]. If the CR sources produce very hard spectra, the energy dependence of the diffusion coefficient in the Galaxy needs to be much stronger than is allowed by the observed CR anisotropy [11]. It should be noted, though, that the CR source spectrum is that of the CR escaping from their sources, the time integral of which could be considerably softer than the spectrum of particles contained in the sources at a given time [12]. On the other hand, recent $\gamma$-ray observations of some shell-type SNRs tend to show soft spectra $(\Gamma>2)$ : RX J1713.7-3946 [13, 14], RX J0852.0-4622 15], RCW 86 [16], SN 1006 [17], Cas A [18, 19], IC 443 [20, 21] and other [22, 23, 24]. This means that the particle spectra, assuming the radiation was produced by hadronic scenario, is also softer than NDSA predictions.

It is known that $\mathrm{CR}$ particles are scattered by magnetohydrodynamic (MHD) waves. These waves, alongside amplified magnetic fields (MFs), may be generated by instabilities invoked by CR streaming, which increases MHD turbulence [4, 25, 26, 27], or directly by pure MHD turbulence induced by propagation of collisionless shock in media [28, 29, 30].

Gyro-resonant instabilities favor the generation of MHD waves that propagate with Alfvén velocity [31], whereas nonresonant instabilities can initially favor nonoscillating and non-propagating modes. If the former dominate, the scattering centers move relative to the plasma, and the inclusion of this so-called Alfvénic drift may soften the spectra so that they could match experimental data [32, 33, 34, 35, 36]. Alternatively, the bulk of Galactic CRs may be produced in a low-efficiency or test-particle mode transferring only $\lesssim 10 \%$ of the SNR energy to CRs [34]. This number is roughly that needed to explain the origin of CRs by acceleration in Supernova Remnants [37.

Given the complexities faced by NDSA theory, in this paper we propose to investigate the diversity of particle spectra that can be produced by young SNR shocks in a test-particle mode, concentrating for simplicity on Type Ia SNRs in this paper. We consider acceleration by both forward and reverse shocks in evolving sphericallysymmetric systems. Many pieces of observational evidence suggest that the reverse shock (RS) may also accelerate particles to high energies. This includes the detection of non-thermal X-rays from the RS of Cas A 38, 39], 1E 0102.2-7219 [40], and RCW 86 [41], as well as radio emission from the RS of Kepler's SNR [42]. CR acceleration at the RS is also supported by theory [43, 44]. In any case, high-energy particles produced by the FS may be re-accelerated at the RS. [45]. The preponderance of observational and experimental evidence, combined with earlier theoretical implications, suggests that it is essential to at least consider 
the effects of both shocks in accelerating particles. The total volume-integrated spectra in such a case will deviate from expectations based on plane-parallel shocks and steady-state systems employing the FS only.

The MF in the shocked interaction region is essential to determining the particle spectra, but is extremely hard to deduce observationally. This has led to the parameterization of the field in different models, which generally involves tying the field to dynamically or kinematically determinable parameters. Our intent is not to study the amplification of the field itself, which is beyond the scope of this paper, but to investigate the particle spectra that are produced under widely used assumptions of the field in the literature. We also explore how the inclusion of Alfvénic drift affects the total particle spectra in SNR. Given the observational data on MF amplification [46, 47] as well as theoretical [4, 25] and numerical [48, 26] results, we consider two cases: a moderate $(75 \mu \mathrm{G})$ and a strongly amplified $(300 \mu \mathrm{G})$ MF. Besides calculating the total particle spectra, we produce wide-band spectral-energy distributions (SEDs), and provide surface brightness profiles in the radio, X-ray and $\gamma$-ray bands.

This work intends to improve upon the present state of the field in many ways (1) We take into account the free expansion phase of the expanding SNR, when the shock velocities are the highest, whereas most (though certainly not all) analyses have totally neglected the initial phase, and started directly with the Sedov phase. (2) Our technique to solve the transport equation, and the corresponding high resolution spherically-symmetric simulations used, guarantee a very high level of accuracy and therefore an accurate rendering of the particle spectra for the chosen
MF and diffusion coefficient. (3) We have taken into account the acceleration of CRs at the reverse shock, as suggested by recent observations. We have shown what observable features in SNRs we may expect if a non-negligible MF permits CR acceleration at the RS to high energies. This has not been explored in such detail before, including spherical symmetry and fully time-dependent cosmic-ray transport. Earlier studies [43] investigated CR acceleration at the RS using an approximate, analytical model [49] and assumed there is no MF amplification at the RS. In particular, they focused on non-linear effects arising from a high injection efficiency in low MF. Other authors [44] concentrated on the study of a specific object, which most probably is a core-collapse SNR, and have not made any general conclusions regarding type-Ia SNRs. (4) Our results therefore present a more complete study of the particle spectra and high-energy emission that is to be expected from type-Ia $\mathrm{SNe}$ with moderate acceleration efficiency.

\section{Cosmic-Ray Acceleration}

We consider a test-particle approach to diffusive shock acceleration of CRs in SNRs [2, 3, 4, 5]. It has been recently [34] shown that a test-particle description is applicable if the CR pressure at the shock is less than $10 \%$ of the shock ram pressure. Therefore, if one limits the amount of energy contained in CRs and the CR pressure at the shock, the acceleration can be treated by independently solving the cosmic-ray transport equation and the hydrodynamic equations of SNR evolution. The cosmic-ray transport equation is a diffusion-advection 
equation in both space and momentum 31]:

$$
\begin{gathered}
\frac{\partial N}{\partial t}=\nabla\left(D_{r} \nabla N-\vec{v} N\right)- \\
\frac{\partial}{\partial p}\left((N \dot{p})-\frac{\nabla \vec{v}}{3} N p\right)+Q
\end{gathered}
$$

where $N$ is the differential number density of cosmic rays, $D_{r}$ is the spatial diffusion coefficient, $\vec{v}$ is the advective velocity given by a 1-D hydrodynamical simulation, $\dot{p}$ are the energy losses, and $Q$ is the source term representing the injection of the thermal particles into the acceleration process given as

$$
Q=\eta_{i} n_{u}\left|V_{s h}-v_{u}\right| \delta\left(r-R_{s h}\right) \delta\left(p-p_{i n j}\right),
$$

where $\eta_{i}$ is the injection efficiency parameter, $n_{u}$ is the number density of plasma in the shock upstream region, $V_{s h}$ is the shock speed, $v_{u}$ is the plasma velocity in the shock upstream region, $r$ is the distance from the SNR center, $R_{s h}$ is the radius of the shock, $p$ is the particle momentum, and $p_{i n j}$ is the momentum of the injected particles.

We assume the thermal leakage injection model [50], where only the particles with momentum $p_{i n j}>\psi p_{t h}$ can be accelerated, with $\psi$ being a multiple of the particle thermal momentum, $p_{t h}$. The efficiency of injection is determined as

$$
\eta_{i}=\frac{4}{3 \sqrt{\pi}}(\sigma-1) \psi^{3} e^{-\psi^{2}}
$$

where $\sigma$ is the shock compression ratio. Although $\sigma$ and $p_{t h}$ are obtained from hydro simulations, $\psi$ remains a free parameter in the model. In our calculations we assume $\psi \simeq 4.45$, keeping $\eta_{i} \simeq 5 \times 10^{-7}$ sufficiently low to stay within the framework of the testparticle approximation. We note that since we are not interested here in the absolute ratio of electron to proton spectra, and lacking secure knowledge of the details of electron injection, as well as the time-scale of thermalization between electrons and protons, we assume that electrons and protons are injected equally and are at the same temperature, i.e. the injected electron to proton ratio, $K_{e / p, i}=1$. Thus, $K_{e / p}$ obtained in our simulations is purely a result of the electron-to-proton mass ratio and corrected for standard ISM abundances.

We impose a free-escape spatial boundary upstream of the FS that should account for escape of the highest-energy particles from the system. We assume that all particles crossing the boundary leave the system, therefore we set the CR number density, $N$, to zero at the free-escape boundary, at $R_{\text {esc }}=2 R_{F S}$, where $R_{F S}$ is the FS radius.

We also assume that the highest energy particles generate MHD waves in the shock upstream region, and thus may amplify the MF [25, 26, 27, 35]. Being scattered by MHD waves, the CR particles undergo Bohm diffusion everywhere inside the escape boundary [51]. Also, the advective velocity in Eq. 1, given the presence of amplified MF, may include the Alfvénic drift of scattering centers and thus may not coincide with the plasma flow velocity [32, 35, 34, 33].

In the current paper we assume spherical symmetry and do not consider the momentum-diffusion term representing stochastic (second order Fermi) acceleration.

The main difficulty for the numerical solution of Eq. 1 is that the diffusion coefficient $D_{r}$ is strongly dependent on the particle momentum $\left(D_{r}=D_{r}(p) \sim p\right.$, in case of Bohm diffusion) and therefore spans a wide range of magnitudes, $\ln \left(p_{\max } / p_{i n j}\right) \approx$ 25. Formally, the spatial grid must resolve the smallest diffusion length defined as $D_{r}\left(p_{i n j}\right) / V_{s h}$, otherwise the modeled ac- 
celeration is artificially slow. The lowestenergy particles have a very small diffusion length, and thus a uniform grid must contain millions (or even billions) of cells which is computationally impossible.

Several approaches are known to date to overcome this numerical difficulty. The socalled normalized grid is used in [52], where a substitution of variables is performed and the spatial coordinates are normalized to the diffusion scale. Another approach presented by [53] consists of introducing a comoving frame, in which the shock is stationary, coupled with a mesh refinement - a fine discretization is used only near the shock region, where the low-energy particles are injected.

Here we use the second approach. We normalize the spatial coordinate in Eq. 1 to the shock radius, introducing coordinate $x=r / R_{s h}$, and then substitute the coordinate $x$ with a new coordinate, $x_{*}$, for which we use a uniform grid when solving Eq. 1:

$$
(x-1)=\left(x_{*}-1\right)^{3}
$$

with Jacobian

$$
\frac{d x}{d x_{*}}=3\left(x_{*}-1\right)^{2}
$$

These simple transformations allow us i) to place the shock at the center of a known cell at any given time and ii) to resolve the shock vicinity for low-energy particles with only a few hundred bins in the spatial coordinate, $x_{*}$. We use parametrizations for the radial dependence of the MF as described in Section 4, and we obtain the hydrodynamical parameters (the plasma flow velocity, density and the shock speed) needed to solve Eq. 1 from 1-D hydrodynamic simulations of the SNR evolution described in Section 3 .
Then, Eq. 1 is solved in sphericallysymmetric geometry using implicit finitedifference methods implemented in the FiPy 54 library modules. We make separate runs for the FS and the RS, using the appropriate coordinate transformations to obtain the highest resolution where injection occurs. Then we sum the results because they are linear and independent. Some of the highest-energy particles may reach the other shock and be re-accelerated there. However, the energy of these particles is already so high, and their number so low, that the poor resolution at the other shock does not visibly affect the results. Finally, we check the CR-to-ram pressure ratio as well as energy containment in CRs a posteriori. In our calculations these values are never allowed to exceed $10 \%$.

\section{Hydrodynamics}

To study the evolution of the SNR shock waves over time, we have performed spherically-symmetric hydrodynamic simulations using the VH-1 code [55], a 3dimensional hydrodynamic code based on the Piecewise Parabolic Method [56]. The simulations are based on an expanding grid [57, 58], which tracks the motion of the outer shock and expands along with it. Thus, a high resolution is maintained right from the start of the simulations, which is essential considering that the remnant size increases by about 6 orders of magnitude during the simulation. The expanding grid also has another advantage - the position of the forward shock is almost stationary on the grid over most of the evolution, which is useful in computing the particle spectrum. Furthermore, the grid expansion is adjusted such that, as far as possible, the shocked interaction region between the in- 
ner and outer shocks occupies most of the grid. Although cooling of the material is incorporated into the simulations via a cooling function, the velocities and densities are such that cooling is not effective and does not play a role.

In order to study the evolution of young $\mathrm{SNe}$ in the ambient medium, we need at minimum two parameters - a description of the density profile of the material ejected in the explosion, and that of the medium into which the $\mathrm{SN}$ is expanding. In this first paper, we consider the evolution of a Type Ia SN, which are thought to arise from the thermonuclear deflagration of a white dwarf. Since the progenitor star is a lowmass star and (presumably) does not undergo significant mass-loss, the medium surrounding it is assumed to be a constantdensity interstellar medium.

The ejecta density structure is more uncertain. However, by comparing spherically-symmetric models of Type Ia SN explosions, it was found [59] that the ejecta structure of a Type Ia SN after explosion can best be represented by an exponential ejecta density profile. This is the profile that we have used in our simulations. Since an exponential introduces an additional dimensional parameter to satisfy a non-dimensional exponent, the resulting SN evolution is no longer self-similar. The SN is homologously expanding, therefore the ejecta velocity simply increases linearly with radius.

The initial conditions then depend on three parameters: (i) The energy in the ejected material, which we take as the canonical energy of a SN explosion, $10^{51} \mathrm{erg}$, (ii) the mass of the ejecta, which we take to be the Chandrashekhar mass, $1.4 \mathrm{M}_{\odot}$, and (iii) the density of the surrounding medium, which we take to be $9.36 \times 10^{-25} \mathrm{~g} \mathrm{~cm}^{-3}$, or

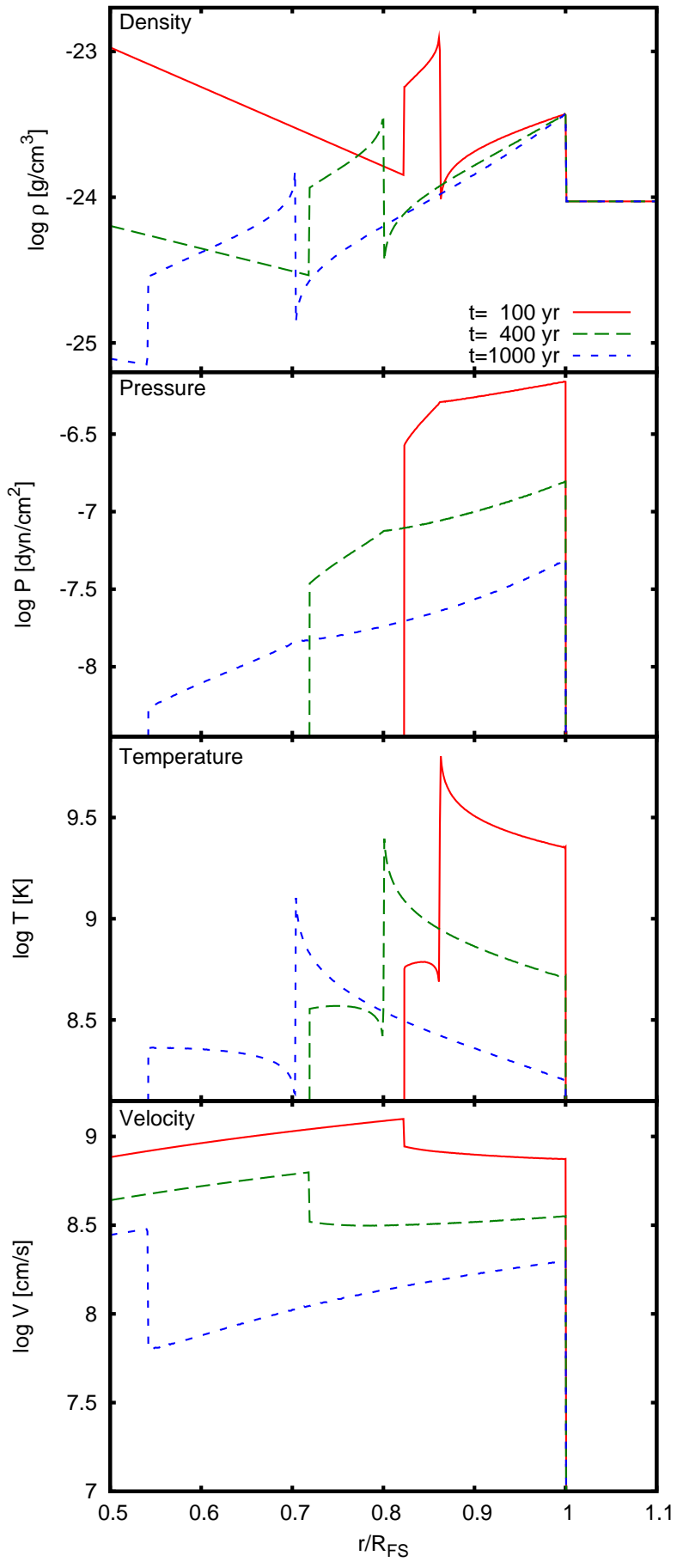

Figure 1: Hydrodynamical parameters of the SNR at different ages. The $\mathrm{x}$-axis is scaled to the radius of the forward shock. 
a number density of about 0.4 for a gas with $90 \% \mathrm{H}, 10 \% \mathrm{He}$, and a trace of other materials.

The supersonic expansion of the SN ejecta into the ambient medium gives rise to a forward shock expanding into the medium, and a reverse shock expanding back into the ejecta in a Lagrangian sense. The two are separated by a contact discontinuity, which separates the shocked ejecta from the shocked ambient medium. Although not captured in our spherically symmetric simulations, in multi-dimensions the decelerating contact discontinuity is always unstable to Rayleigh-Taylor (R-T) instabilities, leading to R-T "fingers" of shocked ejecta expanding into the shocked ambient medium.

The simulation commences with a grid of size $6 \times 10^{-5} \mathrm{pc}$. By the end of the simulation, which is carried on for about 1000 years, it has grown to more than $10 \mathrm{pc}$. The hydrodynamic parameters at the age of 100 , 400, and 1000 years are shown in Fig 1. The outer shock, inner shock and contact discontinuity are all clearly delineated as sharp discontinuities in the density panel (top). After a few hundred years the shocked region occupies more than $90 \%$ of the grid. This resolution is essential for computing the velocity differential across the shock, required in the solution of the cosmic-ray transport equation. Note that, while the velocity varies smoothly across the contact discontinuity (CD), and the pressure shows a slight change in slope, there is a dramatic change in density across the CD. The shocked ejecta reach a maximum density on the inner side of the contact discontinuity, while the shocked ambient medium reaches a minimum on the outer side. The temperature therefore reaches a maximum at the CD for the shocked ambient medium. This distinguishes it from the structure obtained for a power-law profile expanding into the ambient medium, as would be more appropriate for a Type II or core-collapse SN [59].

Finally, the numerical solution of the hydrodynamic equations is mapped onto the spatial coordinate $x_{*}$ (cf. Eq4) in which we have written the particle transport equation (Eq. 11). The shocks, which are invariably smeared out in the diffusive hydrodynamic calculation, are re-sharpened by interpolation toward a point-like jump in density, flow velocity, and pressure. This is necessary to maintain a realistically fast acceleration at $\mathrm{GeV}$-to- $\mathrm{TeV}$ energies.

\section{Magnetic Field and Models Setup}

\subsection{Magnetic Field Amplification at SNR shocks}

The strength of the magnetic field in the SNR is one of the crucial parameters for CR acceleration, particularly so in the shock regions. Since we assume Bohm diffusion here, the MF determines the mean free path of the particle before it is scattered. For higher MF a particle will be more quickly accelerated and it can attain a higher energy before it may escape to the far-upstream region of the forward shock. Therefore, the MF determines the maximum energy of both protons and electrons, the latter in addition suffering energy losses determined mainly by the same MF.

The actual mechanism of MF amplification (MFA) is a subject of ongoing research. Despite recent progress [60, 26, 35], it is not yet clear what mechanism (or most probably a combination of mechanisms) is responsible for induction of MHD turbulence on scales needed for the particle acceleration (for a recent review see [61] and references therein). Due to the uncertainty in 


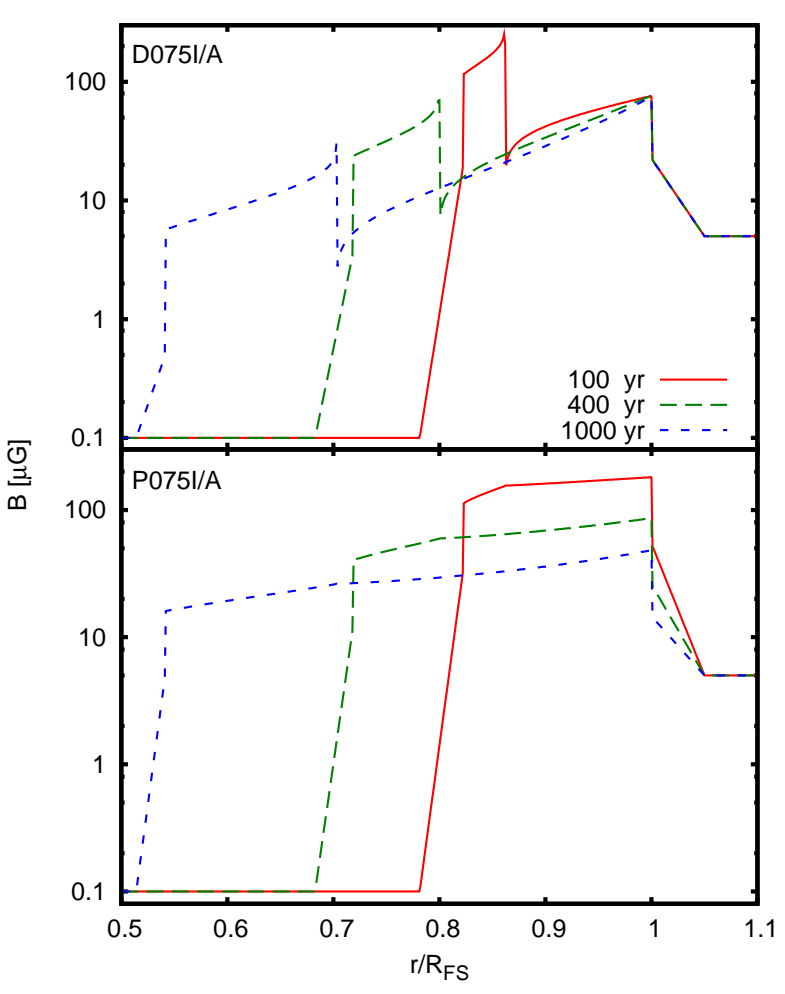

Figure 2: Magnetic field profiles in the SNR for D and $\mathrm{P}$ type models at different ages.

the dominant mechanism of MFA, and the difficulty of taking into account the complexiety of the MFA process, the magnetic field in SNRs is often phenomenologically parametrized. If the MF is assumed to be frozen in, the energy density of the field may correlate with the thermal and relativistic particle energy densities. Here we investigate the impact of widely used MF profiles on the spectra of accelerated particles. We introduce a set of simple scalings which describe the temporal and spatial evolution of the magnetic field in a SNR. The models are listed in Table 1 and are explained below in subsection 4.2 .

Prompted by recent high resolution Xray observations, which claim to detect nonthermal emission from the reverse-shock re-
Table 1: Nomenclature of MF models used in this study.

\begin{tabular}{|c|c|c|}
\hline $\mathrm{MF}$ Profile & Density & Pressure \\
\hline $75 \mu \mathrm{G}$ & D075I & P075I \\
\hline + Alfvénic drift & D075A & P075A \\
\hline $300 \mu \mathrm{G}$ & D300I & P300I \\
\hline + Alfvénic drift & D300A & P300A \\
\hline
\end{tabular}

gion of some SNRs [38, 39, 40, 41], we have chosen to consider particle acceleration at the reverse shock in addition to that at the forward shock. The major argument against the ability of the RS to accelerate particles is that an exceptionally low MF exists in the ejecta, a theoretical argument obtained under the assumption that the magnetic flux at the surface of progenitor star is conserved during the expansion of the SNR. However, the detection of non-thermal Xrays from the RS implies that there may be some processes acting against rapid dilution of the MF, and that a large MF at the reverse shock can exist. These processes may be the fluid-dynamical MHD turbulence in the ejecta and CD region - the initial acceleration of CRs, while the MF was high enough, with subsequent streaming through a confined ejecta region (accelerated CRs do not leave the upstream region of the RS as easy as that of the FS). It is also not clear whether the MF in the ejecta, to be scaled according to flux conservation, must be the field at the surface of SN progenitor. Finally, the fact that shocks in SNRs are collisionless implies the existence of a finite $\mathrm{MF}$ which mediates the collisionless shock. Additional justification for the presence of MF at the RS can be found in [44]. 


\subsection{Parametrizations of Magnetic Field Profiles}

The actual amplification of the magnetic field is a complicated, turbulent process that is not well understood. Many published studies of particle acceleration in SNRs assume that the MF is proportional to the density of the plasma (e.g., [33, 44, 62, 52]). An alternative approach is generally adopted in models of $\mathrm{SNe}$ radio emission, where the MF energy density in the remnant is proportional to the thermal energy density, and thereby the gas pressure [63] (for review see [64]). Following these scalings, we set up $8 \mathrm{MF}$ models, 4 'D' models following the density profiles and 4 'P' models scaling with the square root of pressure profiles. All our models are time dependent, but the matching at the FS is different for the two classes of MF models. $\mathrm{D}$ models assume a constant value of the MF at the FS, $B_{0}=B_{F S}$, and only the scaling inside the SNR proportionally follows the time-dependent density distribution, $B(r, t)=B_{0} \rho(r, t) / \rho\left(R_{F S}, t\right)$. In contrast, $\mathrm{P}$ models assume that the MF at the FS and inside the SNR both evolve with time according to the square root of pressure distribution, $B(r, t)=\sqrt{C 8 \pi P(r, t)}$. Here, $\rho(r, t)$ and $P(r, t)$ are the density and the pressure profiles of the plasma inside the SNR, and $C$ is a proportionality constant chosen so that $B\left(R_{F S}, t_{m}\right)=B_{F S}$, where $t_{m}=500$ years is the mid-time of simulations. We assume two possible values of the MF at the SNR forward shock, $B_{F S}=75 \mu \mathrm{G}$ and $B_{F S}=300 \mu \mathrm{G}$. These values are taken in accordance with average limits given by the observational data on the MF for young SNRs in X-rays [47] and gamma-rays [46]. The proportionality constant, $C$, is rather small in both cases, $C=0.19 \%$ for $B_{F S}=75 \mu \mathrm{G}$ and $C=3 \%$ for $B_{F S}=300 \mu \mathrm{G}$. The amplified value of the MF at the $\mathrm{RS}$ varies between $\simeq 5$ and $\simeq 30 \mu \mathrm{G}$ depending on the age and model. Even if we start from a rather diluted $\mathrm{MF}$, this requires amplification factors of $\simeq 50-300$, which are not unreasonable in CR-invoked or fluid-mechanical turbulent amplification.

The hydrodynamic profiles allow for MF scaling only inside the shocked region. Generally one can expect that the MF amplification processes operate throughout the entire shock precursor region [26], and thus the MF should show a smooth transition from far-upstream values to amplified values, which are then compressed to the given amplitudes at the shock $\left(B_{F S}\right.$ in D models and $B\left(R_{F S}, t\right)$ in $\mathrm{P}$ models). We therefore assume that the MF falls off to the interstellar field $(5 \mu \mathrm{G})$ at the distance of $5 \%$ of the FS radius ahead of the FS, and down to a very small ejecta field $(0.01-0.1 \mu \mathrm{G})$ at the distance of $5 \%$ of the $\mathrm{RS}$ radius toward the interior [44]. This transition may be explained by an exponential decrease in the number density of high energy particles, which may cause MF amplification. In general, one can also expect that the diffusion coefficient becomes larger than Bohm diffusion, albeit still much lower than the Galactic one due to the enhanced flux of CRs. The transition to the Galactic diffusion occurs at the escape boundary, where the concentration of CRs tends to zero. Here we assume that Bohm diffusion operates up to escape boundary, $R_{\text {esc }}$. In our simulations, we assume that in young SNRs the MF is fully isotropic on account of efficient turbulent field amplification, therefore the MF compression ratio is $\sigma_{M F}=\sqrt{\left(1+2 \sigma^{2}\right) / 3}=$ $\sqrt{11}$, where $\sigma$ is the gas compression ratio. The distributions of the MF for different 
model types and different times are given at Fig 2 .

\subsection{Alfvén Drift of Scattering Centers}

As noted earlier, we also investigate the influence on the particle spectra of a possible drift of the scattering centers upstream of the shocks. Resonant streaming instabilities in the precursor region of the shock generate outward-moving Alfvén waves [26] that may have a significant phase velocity in the presence of amplified MF. As particle are scattered on excited MHD waves, the Alfvén velocity of the waves may effectively change the compression ratio felt by the particles, and the spectrum becomes noticeably softer [65, 32, 35, 33]. In cases involving an Alfvénic Mach number $M_{A} \lesssim 10$, the test-particle slope is softer, $s<2$, than the classical solution [34]. It should be noted that non-resonant instabilities often involve turbulence with very small phase velocity [25] for which Alfvénic drift is ignorable. Which type of instability dominates and what the turbulence properties are in the nonlinear regime is the subject of ongoing research [66, 67, 68]. Here we parametrically study the impact of Alfvénic drift. In half of our models we introduce Alfvénic drift in the upstream regions of both shocks assuming that the effective velocity of the scattering centers equals the Alfvén velocity, $v_{A}=B_{F S} / \sqrt{4 \pi \rho_{I S M}}$, plus the upstream gas velocity. In the downstream region, we assume that the scattering centers are isotropized and there is no Alfvénic drift. We mark the models including Alfvénic drift with "A " while the others are marked with "I".

\section{Results and Discussion}

In this section we present our main results. We discuss spectra of particles ac- celerated at the reverse and the forward shocks, analyze their properties and evolutionary differences, and explore the intrinsic differences in particle spectra imposed by different parametrizations of the MF and how the Alfvénic drift of scattering centers affects particle spectra. Finally, we calculate the emission from the particles in SNR and discuss the resulting observable properties which could be compared to the experimental data.

\subsection{Particle Spectra}

\subsubsection{Forward vs. Reverse Shock}

The non-thermal spectra of accelerated particles are characterized by a power-law index and a cut-off energy. The powerlaw slope according to DSA theory depends solely on the compression ratio of the plasma flow (velocity jump across the shock). At the location of the shock, where the plasma is compressed, particles adiabatically gain energy and then lose it everywhere in the expanding flow. In the evolving system, the average compression ratio should be considered. Our hydrodynamical simulations were performed with sufficiently high resolution that the deviations of the shock compression ratio do not affect the spectral slope through the entire evolution. The compression ratios in our simulation are $\sigma_{F S}=4.01 \pm 0.09$ and $\sigma_{R S}=3.97 \pm 0.11$, for the FS and the RS respectively.

In the models without Alfvénic drift, the particles accelerated at the FS obey the classical simple power-law distributions with index $s \simeq 2$ up to the energy, at which the escape of the particles from the system (for protons) or the energy losses (for electrons) inhibit further acceleration. As predicted by DSA [69], for protons $E_{\text {max }, p} \sim$ $V_{s h}^{2} B_{0} t$. Therefore, the increase of $E_{\max , p}$ due to system expansion (higher energies 

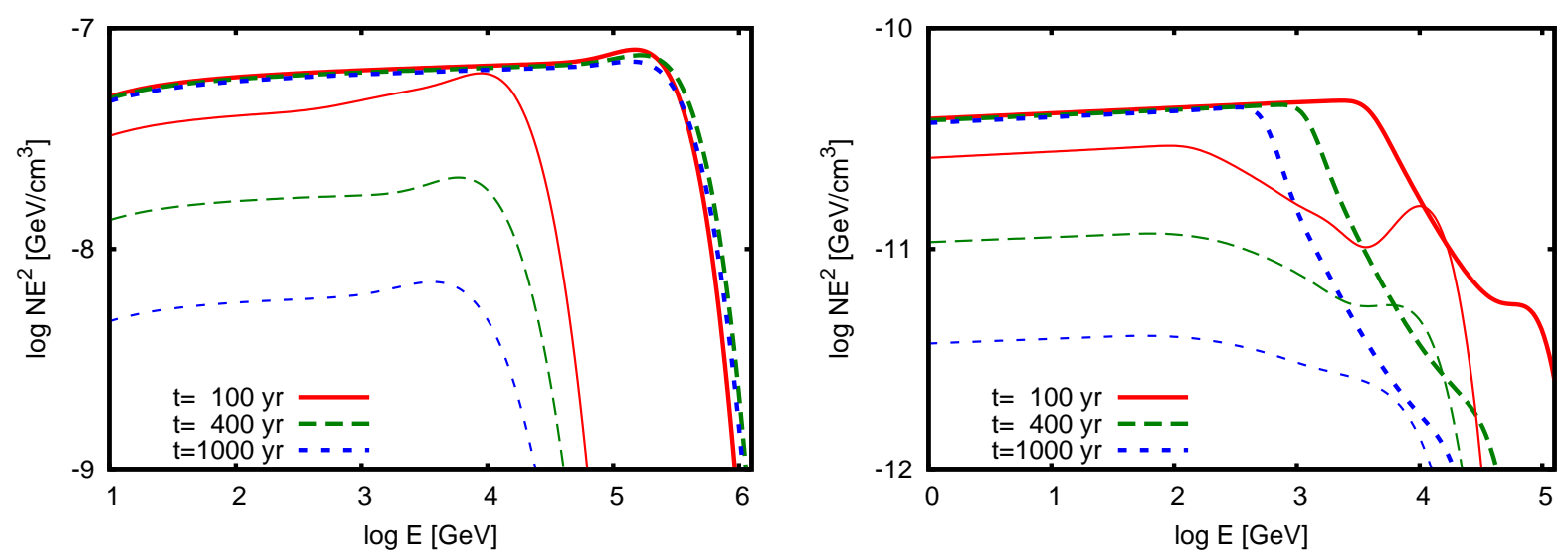

Figure 3: The FS (thick lines) vs. the RS (thin lines) contributions to the spectra of protons (left) and electrons (right) for different SNR ages.

are needed to leave the system) is eventually compensated by the decrease of $V_{s h}$, so $E_{\text {max }, p}$ decreases after reaching a maximum. This is illustrated by the time evolution of FS proton spectra in Fig. 3 (left), which shows the D300I model, in which $B_{0}$ is kept constant, thus isolating $E_{\max , p} \propto V_{s h}^{2} t$. For electrons, $E_{\text {max }, e}$ evolves in the same manner up to the time when the energy gain per cycle no longer exceeds the energy loss per cycle due to synchrotron radiation, i.e, $t_{a c c}=t_{r a d}$. A second, typically lower, characteristic energy, $E_{r a d}$, is approximately set by equality of the radiative-loss timescale with the age of the remnant. Above $E_{\text {rad }}$, the electron spectra assume a quasi steadystate, changing slowly on account of the evolution of the gas-flow profiles. Besides, above $E_{r a d}$ the electron spectra steepen by one power approximately (Fig. 3, right).

The ability of the RS to accelerate particles depends on the MF configuration at the location of the RS. With the MF profiles assumed here and discussed above, it is observed that the RS is capable of accelerating particles to sufficiently high energies and intensities to contribute to the total volume- integrated particle spectra. These results are at least consistent with many of the observations noted above. In order to obtain the volume-integrated spectra, $N(E)$, one performs an integral of the spatially differential particle distribution, $N(x, E)$, over the entire volume of the simulation grid up to $R_{e s c}=2 R_{F S}$ :

$$
N(E)=4 \pi R_{F S}^{3} \int_{0}^{2} N(x, E) x^{2} d x
$$

where $x=r / R_{F S}$. In all plots featuring particle spectra we show the quantity $N=N(E) / 4 \pi R_{F S}^{3}$. In the absence of Alfvénic drift, the RS spectra show slopes similar to those at the FS, with the exception of some hardening at higher energies. The maximum energies for protons are lower than at the FS on account of a lower MF strength, which allows particles to escape the acceleration region at lower energies. In contrast, the maximum energies for electrons are nearly as high as at the FS mainly because a weaker MF also reduces the rate of radiative losses of electrons. The intensity of the volume-integrated RS spectra falls with time relative to the FS spectra 
as the number of injected particles becomes smaller. Also the ratio of the RS surface to FS surface decreases with time, which means fewer particles that could be potentially injected cross the RS.

The stronger spectral hardening and more significant bump at high energies make the RS volume-integrated spectra different from those of the FS. The spectral hardening is most visible at early evolutionary epochs and gradually disappears with time for both shocks, but at a somewhat faster rate for the FS. The bump in the forward and the reverse shock spectra is located close to $E_{\text {max }}$, which is beyond $E_{\text {rad }}$ for electrons. The presence of these spectral features and their evolution can be explained by the geometry of our sphericallysymmetric system.

In order to get volume-integrated spectra we integrate over the entire simulation domain, so both upstream and downstream regions contribute to the volume-integrated spectra. The volume-integrated upstream spectra usually have hard slopes, $s \simeq 1$, and the intensity of the upstream spectra around $E_{\max }$ is comparable with that of the downstream spectra. Thus, in spectra integrated over both upstream and downstream regions one can see the contribution from the hard upstream spectra in highenergies close to $E_{\max }$, whereas the contribution of low-energy particles is negligible. Besides, the $E_{m a x}$-region of the upstream spectra exhibits a bump which appears on account of the exponential decrease of the MF in the upstream direction. As a result, the diffusion time of particles located close to the shock is longer than that of particles further away from the shock. Therefore, the recently accelerated particles around $E_{\max }$ are accumulated near the shock in the upstream re- gion. The spectral hardening and the bump is strongly visible in the RS spectra (Fig. 3, right) because the volume of the upstream regions of both shocks are significantly different in the spherically-symmetric geometry assumed here, contrary to the case of plane-parallel shocks. This also explains the different time evolution of the spectral features. The upstream region of the FS expands significantly faster than the upstream region of the RS, thus the CR number density in the upstream region of the FS decreases faster and contributes less to the total volume-integrated spectra. We note the presence of a peculiar wiggle in the electron spectra. The MF in the upstream region is lower than downstream, resulting in weaker synchrotron losses for the electrons and therefore $E_{\text {rad }} \simeq E_{\text {max }, e}$. Consequently, the upstream electron spectra have a significantly higher intensity near $E_{\text {max }, e}$ than the downstream electron spectra. Therefore, in the total volume-integrated spectra the bump stands out beyond $E_{\text {rad }}$ in the $E_{\text {max }, e}$ region. For protons, $E_{\text {max }, p}$ in the upstream and downstream spectra nearly coincide, and a small bump appears near $E_{\text {max }, p}$ in the total volume-integrated proton spectra. Again, due to the faster decrease in the CR number density ahead of the FS with time, the feature is more prominent in the RS spectra.

\subsubsection{Influence of Magnetic Field Profiles and Strengths}

Most important for DSA is the configuration, strength, and evolution of the MF at the location of the shocks and their precursor regions. Since here we adopted similar profiles of $\mathrm{MF}$ in the precursor regions of the shocks, only a different time evolution of the MF strength at the location of the shocks may change the shape of the volume- 


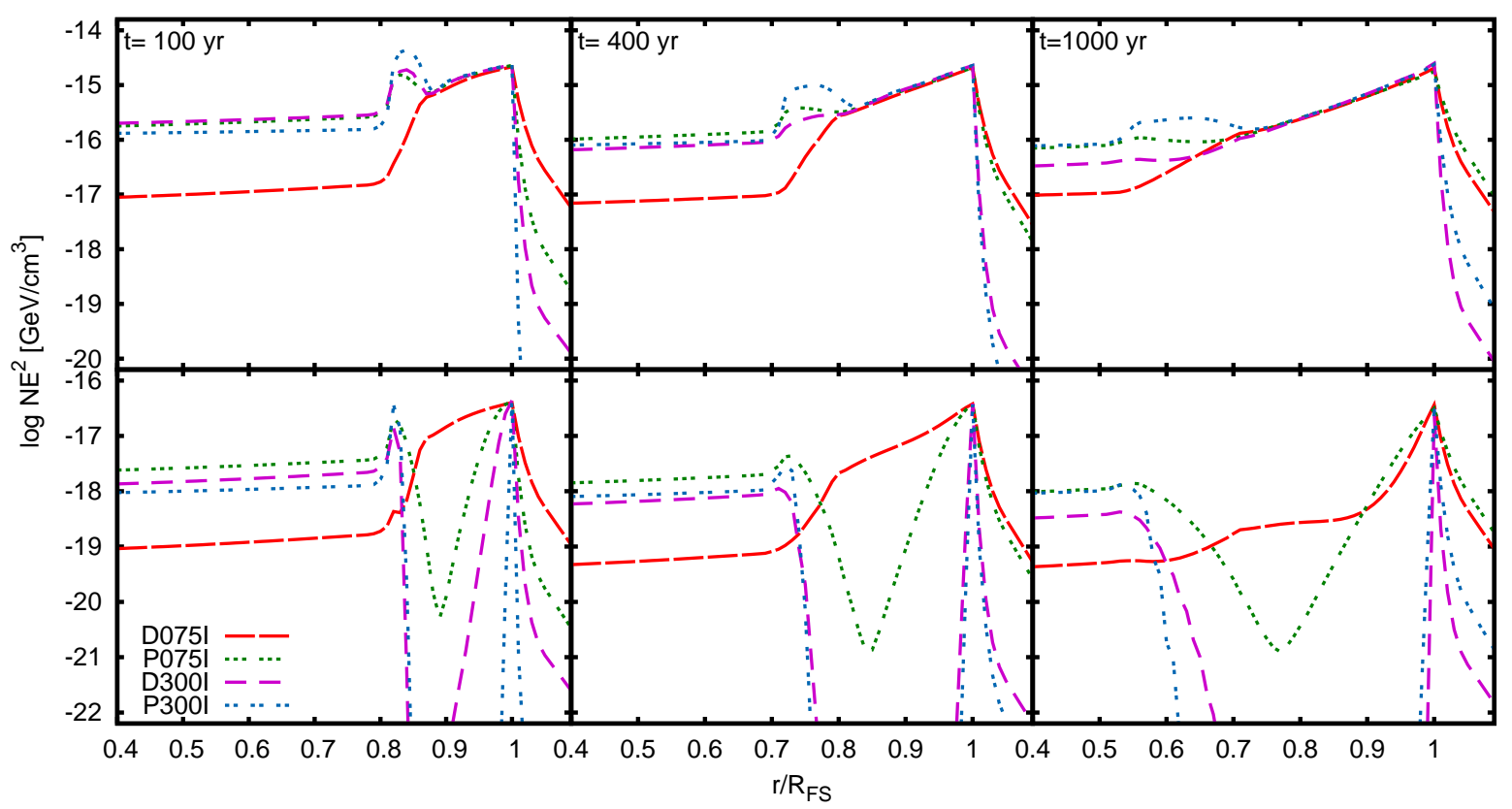

Figure 4: The time evolution of the spatial distribution of protons (top row) and electrons (bottom row) at energy of $20 \mathrm{TeV}$.

integrated spectra. It was noted, however, that the differences between models in the spectra of particles created by the RS and the FS are marginal, in fact only $E_{\text {rad }}$ and $E_{\text {max }}$ truly depend on the MF strengths. While for protons a higher MF would mean a higher $E_{\max , p}$, for electrons this would mean a lower $E_{r a d}$ and $E_{m a x, e}$ due to radiative losses.

The MF profiles in the SNR interior affect the radial distribution of $\mathrm{CR}$ particles and their subsequent emission, especially so for leptons. In Fig 4 we plot the evolution of the radial distribution of protons and electrons at the energy of $20 \mathrm{TeV}$, which could be a characteristic energy of the particles producing high-energy emission, discussed in more detail in subsection 5.2.2. We use models without Alfvénic drift as it does not change the distribution significantly it would only slightly reduce the intensity at the FS. One can clearly see that electrons suffer significant losses in the shocked region where the MF is high. The number density of protons falls nearly exponentially from the FS towards the CD. There is a noticeable peak in the CR distributions near the RS. Whereas electrons peak exactly at the RS, protons are accumulated between the RS and the CD. At 100 years, the $\mathrm{CR}$ number density close to the $\mathrm{RS}$ is even higher than at the FS. At 400 years the peak is still clearly visible, however already at 1000 years only a minor bump is observed. In the ejecta region, where the MF is low, both particle species show nearly uniform distributions. Although the D075I model follows the trends described above, it is a clear outlier. The cut-off energy of particles accelerated by the RS in this model is around $4 \mathrm{TeV}$, so one finds at $20 \mathrm{TeV}$ almost no particles from the RS. This ex- 


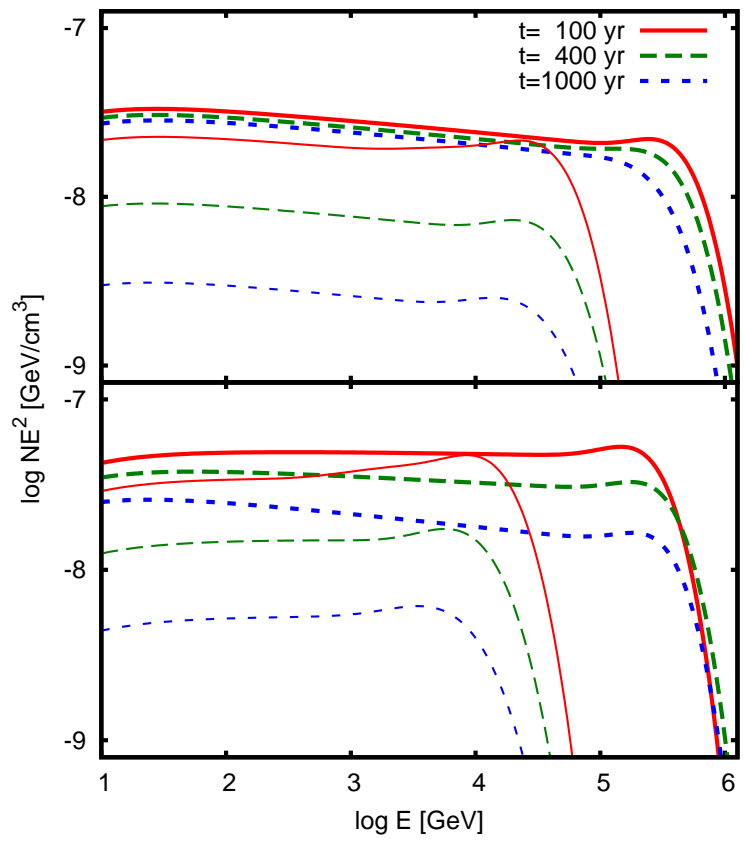

Figure 5: The spectra of the FS (thick lines) and the RS (thin lines) for different SNR ages in P300A (top) and D300A (bottom) models.

plains the absence of a peak near the RS and the low CR number density in the interior of the SNR. Besides, the electrons have suffered little energy losses in the initial phases of SNR evolution $\left(t<t_{\text {rad }}\right)$ compared with other models (remember that P075I model have initially a high MF).

\subsubsection{Impact of Alfvénic Drift}

We have explored the influence of Alfvénic drift on the particle spectra of the SNR. Interesting is that it affects our models in a different way during the SNR evolution. We observe that the FS and the RS spectra in $\mathrm{P}$ models are noticeably softer during all evolutionary times. This is because in $\mathrm{P}$ models the Alfvénic Mach number, $M_{A}$, is almost constant for both shocks during the evolution. The MF strength, $B$, evolves as the square root of the pres- sure, $\sqrt{P}$, and hence roughly scales with the shock velocity. Therefore, spectra show nearly the same softening at all times as shown at the top of Fig. 5. Contrary to P models, in $\mathrm{D}$ models the effect of Alfvénic drift is different for the FS and the RS. At the FS $B$ is kept constant during the evolution, and $M_{A}$ is very large at early times because the Alfvén velocity, $v_{A}$, is small compared to the shock speed. At later times $v_{A}$ remains constant, but the shock speed drops, and so $M_{A}$ becomes small. This implies that the softening of the FS spectra in D models was small at early times and increased during the evolution of the SNR. In contrast to the FS, $B$ is evolving at the RS as density, $\rho$. The initially large ejecta density caused a high Alfvén velocity $\left(v_{A} \sim \sqrt{\rho}\right.$ in $\mathrm{D}$ models $)$ and rather small $M_{A}$, though larger than at the FS, which caused some softening of the spectra. Over time $\rho$ dropped significantly, and therefore $M_{A}$ became large. Therefore, the softening of the RS spectra in D models is stronger at earlier times and almost not noticeable at later times (Fig. 5, bottom). Obviously, all these effects and characteristics are stronger in models with high MF strength.

\subsubsection{Total spectra from both shocks}

The shape of the total volume-integrated spectra depends on the SNR age on account of the changing importance of the contributions from the forward and reverse shocks. For very young Type Ia SNRs, and keeping in mind the MF profiles assumed herein, a significant contribution to the total spectrum arises from the RS. However, already after a few hundred years the bulk of particles is provided by the FS. Nevertheless, particles accelerated at the RS continue to create peculiar spectral features up to late times of the SNR free expansion phase (see 
Fig. 61). Added to this is the different impact of Alfvénic drift on the spectra produced with different MF models and the time evolution of this impact, especially in $\mathrm{D}$ models. The total spectra are very different from a single-shock test-particle solutions as seen at Fig. 6. We plotted 6 out of 8 models because D075A and P075A models show similar shapes as D075I and P075I models, apart from being slighter softer.

At 100 years the contribution of the RS to the total spectra is significant in all models. With age this contribution decreases. After 400 and 1000 years, the proton spectra in $\mathrm{P}$ models look like broken power laws or vary gradually, much smoother than exponentially cut-off. Since the RS proton spectra in D models have a somewhat smaller cut-off energy and are harder than the FS spectra, if Alfvénic drift is assumed, the RS maintains a significant contribution to the total spectrum until late epochs. Because of it and because of the bump contributed by the upstream region of the FS, the proton spectra of D models show some small-scale concavity at high energy through all times, however at later times it is shallower and broader (bumps in both RS and FS spectra become less significant).

The total electron spectra (Fig. 6, bottom row) also have interesting features. On account of energy losses, the contribution of the RS in the cut-off region is stronger. This creates either small-scale concavity or a broken power-low in the loss-affected region (between $E_{r a d}$ and $E_{\max , e}$ ). The stronger the contribution from the RS is, the more pronounced a concavity is observed. When the contribution of the RS is mild, then one sees a broken power-law. In some models, (i.e., D300I and D300A), the shallow concavity in the loss-affected region is visible throughout all late times. It is striking that the electron spectra in single-shock NDSA simulations [70] have similar concavity in the loss-affected spectral band. Additionally, one can note that near $E_{\text {rad }}$ the spectra of $\mathrm{P}$ models are smoother than the spectra of $\mathrm{D}$ models.

We must make an important remark concerning particle spectra. The plotted spectra are integrated over the entire simulation domain. However, one finds (5.2.1) that the main contribution to the emission is done by the particles confined inside the remnant. These particle spectra are typically softer at high energies because they do not include the hard upstream particles. For illustration see the thin lines of D075I models in Fig. 6, which show separately SNR-confined (downstream) particles and peaked at high energies upstream particles. Only inverse Compton emission because of low MF in the upstream region can benefit from the upstream particle population.

\subsection{Particle Radiation}

We consider three basic non-thermal emission processes, synchrotron and inverse Compton (IC) emission of electrons, and neutral pion decays originating in collisions of $\mathrm{CR}$ protons with protons at rest. The synchrotron emission is calculated according to [71]. We consider only the CMB photon field for the IC scattering. Therefore, we may use a simple rescaling of the synchrotron emission to IC scattering [72, 73]. We calculate the gamma-ray emission produced by pion decays according to the procedure described in [74]. Thermal emission is not considered. However, we made estimates that even assuming instant equilibration the maximum of the thermal emission does not exceed $10 \%$ of the synchrotron emission at the respective energy for all considered ages. In particular, at $3 \mathrm{keV}$ the 


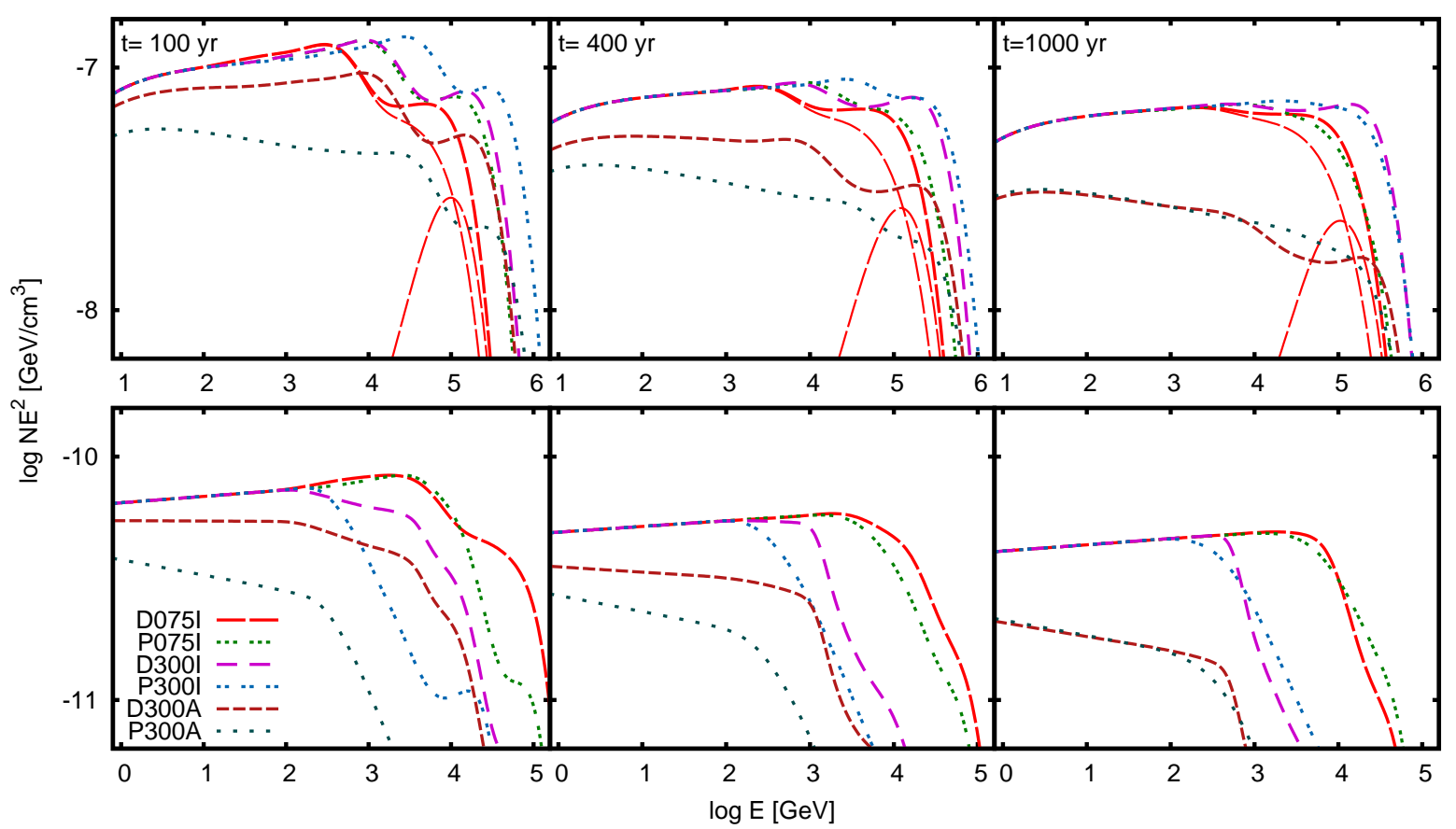

Figure 6: The time evolution of volume-integrated proton (top row) and electron (bottom row) spectra for different models and SNR ages. Thin lines for the D075I model in the top row display the downstream and upstream components of the proton spectra.

thermal component may comprise $\simeq 3 \%$ of the X-ray emission of the models considered here. The reader should be aware though that we used the same injection efficiency for electrons and protons which results in high amplitudes of leptonic emission. If the electron injection efficiency is lowered significantly, below $3 \mathrm{keV}$ the brightness profiles may be affected.

\subsubsection{Radiation Spectra}

The radiation spectra show diversity during SNR evolution (see Fig. 7). In general, the radiation spectra are much smoother than the parent particle distributions. The leptonic spectra show broad features, which are the result of energy losses experienced by electrons during their acceleration history. A noticeable feature of the leptonic spectra is concavity, which appears in dif- ferent models at different times. At 100 years it is strongly visible in P300I and P300A models, but in D075I and D075A it is nearly invisible. Later, at 400 years, it appears in D300I and D300A models while it disappears in D075I and D075A, and it starts to vanish in P300I and P300A models. At 1000 years it is visible only in D300I and D300A models. The concavity reflects the change in electron slope on account of the contribution of the RS between $E_{\text {rad }}$ and $E_{\max , e}$. It interesting to see that the volume-integrated synchrotron and IC spectra have different shapes. While the synchrotron emission comes mainly from the shocked region, a significant fraction of IC emission comes from the ejecta and upstream region with low MF, where the electron spectrum is different. The pion-decay 

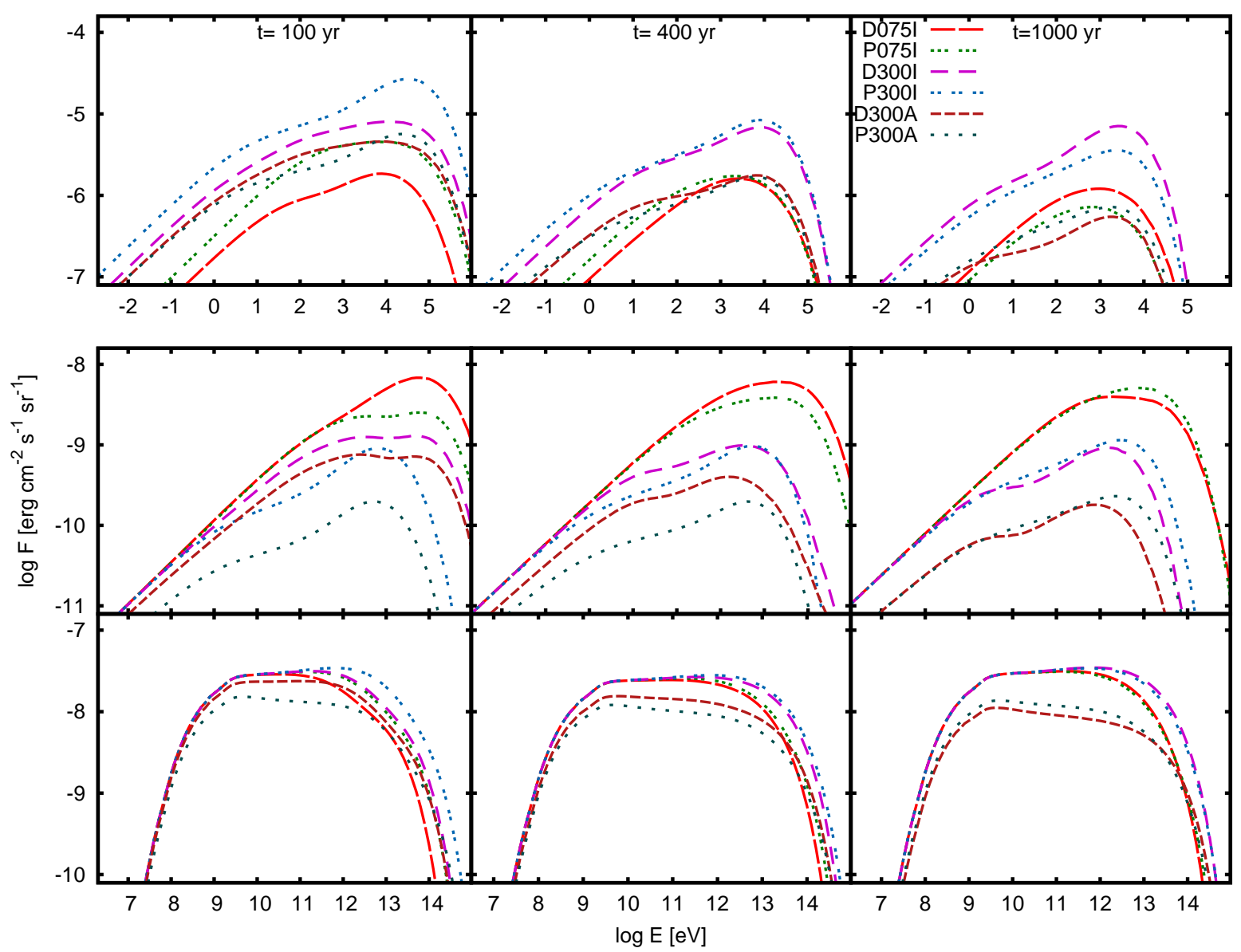

Figure 7: The time evolution of volume-integrated emission from the SNR due to synchrotron (top row), inverse Compton (middle row) and pion-decay (bottom row) for different models and SNR ages.

spectra are much smoother than their leptonic counterparts and can be characterized by a power-law with a very gradual and broad cut off at relatively low energy between a few $100 \mathrm{GeV}$ to a few $\mathrm{TeV}$. This makes the spectra rather soft in the energy band observed with modern Cherenkov telescopes. The pion-decay emission comes mainly from inside the SNR, with a small amount of upstream contribution.

\subsubsection{Brightness Profiles}

To permit morphological studies of SNR at different ages, we computed radial inten- sity profiles at characteristic wavebands for current instrumentation (radio @1.4 GHz, X-rays @3 keV, gamma-rays @1 TeV). In Fig. 8 we separately plot the emission of leptonic and hadronic origin. The profiles are normalized to the emission maximum along the SNR radius. The absolute intensity may be obtained from the spectra given at Fig. 7 , We plot here "I" models only. Their "A" counterparts show similar profiles, but the intensity of the FS is a bit lower, making the profiles look somewhat flatter.

It is astonishing to see how the brightness profiles undergo severe changes with time. 

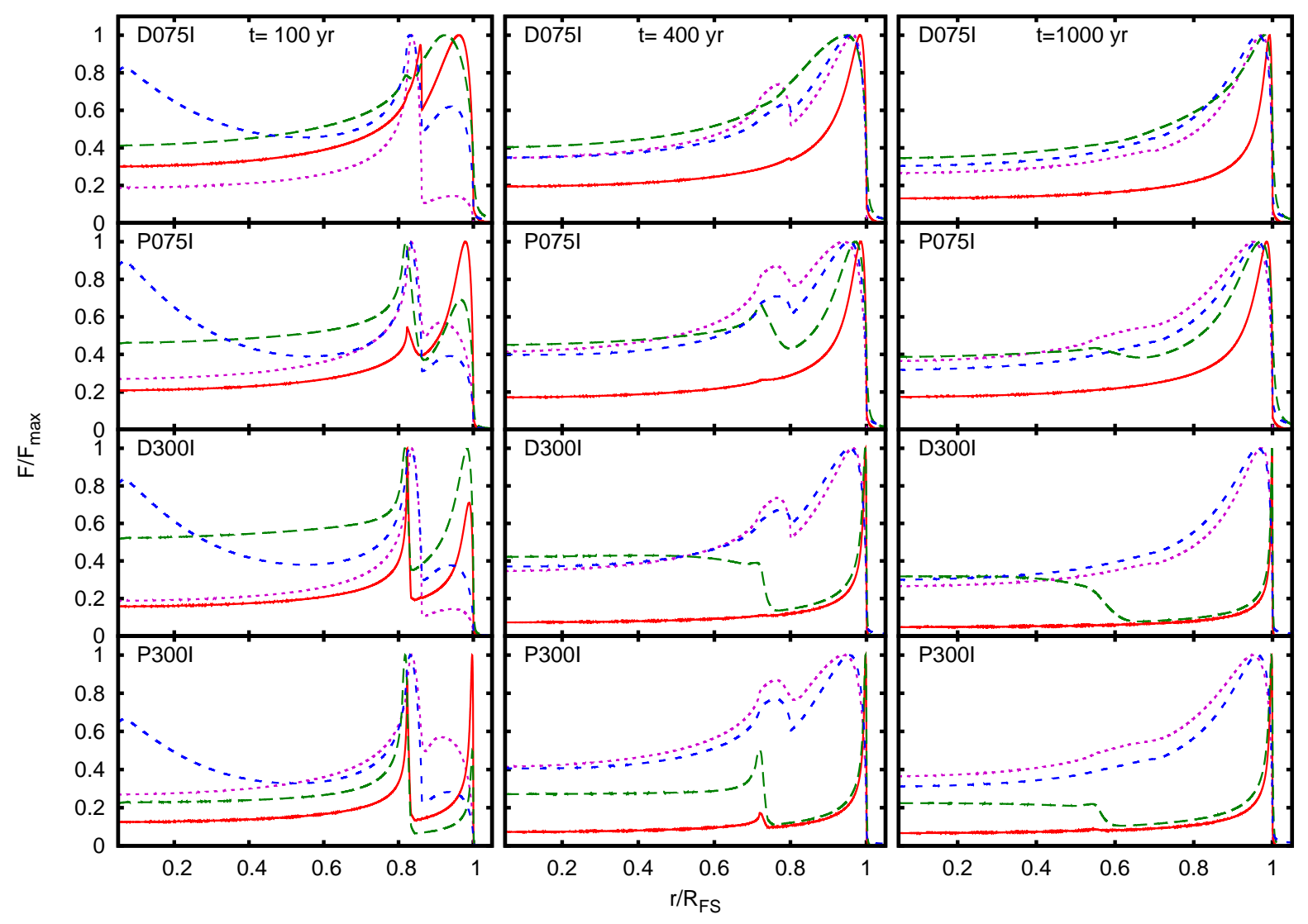

Figure 8: The radial intensity profiles of the SNR in different models at different wavelength and ages. Radio @1.4 GHz (dotted-pink), X-rays @3 keV (solid red), IC @1 TeV (long-dashed green), pion-decay @1 TeV (dashed blue).

At 100 years both shocks are bright and visible in all bands except for the FS in radio waves, which are emitted by low-energy electrons. The contribution of the RS to the total emission is high at the initial stages. The low-energy electrons cannot propagate far from their point of origin, and therefore the relatively high injection rate at the RS in the early phase renders it much brighter than the FS in radio band. Pion-decay gamma rays are copiously produced in the ejecta region on account of the high target density there. At 400 years the contribution of the RS region becomes less prominent, but is still visible. Only in synchrotron X- rays (and IC in D075I/A models) the RS becomes insignificant. The $3 \mathrm{keV}$ synchrotron $\mathrm{X}$-rays are created by electrons of rather high energy $(\sim 13.5 \mathrm{TeV}$ for $B=300 \mu \mathrm{G}$ and $\sim 27 \mathrm{TeV}$ for $B=75 \mu \mathrm{G}$ ). At this age the RS is no longer able to boost electrons up to these energies. The electrons accelerated at the FS suffer from losses on their way to the SNR interior, and therefore the synchrotron emission becomes noticeably filamentary, especially in high-MF models. A characteristic feature develops in IC emission of the models with high MF. A plateau of high intensity appears in the ejecta region and a low intensity between 
the two shocks. In the shocked region, highenergy electrons are few on account of severe energy losses in the high $\mathrm{MF}$, so the IC emission is suppressed. At the same time, the electrons that propagated into the ejecta region, where the MF is low, accumulate there and account for bright IC emission. This trend continues with age, and after 1000 years we observe a bright plateau of IC emission from the SNR center, while the FS appears very filamentary. There is almost no trace of emission from the RS after 1000 years in the other wavebands. X-ray synchrotron emission is seen as a thin filament near the FS, especially in the models with high MF.

\section{Conclusions}

We have studied particle acceleration by both forward and reverse shocks in young type-Ia Supernova Remnants. Rather than starting with Sedov models, as is most commonly done, we used gas-flow profiles in the ejecta-dominated phase, derived from 1-D hydrodynamical simulations, to solve the cosmic-ray transport equation in a testparticle approach. We analyzed how different phenomenological parametrizations for the magnetic-field distribution and strength affect the maximum energy and spatial distributions of accelerated particles. Additionally, we have explored the influence of possible Alfvénic drift of scattering centers on the total particle spectra in the upstream region of both SNR shocks and find our results for the forward shock in agreement with previous findings [32, 35, 34]. We investigated the properties of the resulting non-thermal radiation, i.e., its spectra and the spatial distribution of the intensity at characteristic wavelengths.
We have demonstrated that it is important to account for the contribution of the $\mathrm{RS}$ to cosmic-ray particle population in the initial 400 - 600 years of SNR evolution, given the widely used parametrizations of the MF that are assumed here. At that time the RS is able to accelerate particles up to very high energies, and the number of accelerated particles is comparable to that at the FS. This is well visible in volumeintegrated particle spectra, the distribution of high-energy particles in the SNR, as well as in the morphology of particle radiation. The significance of the RS contribution falls with time. Although the RS contribution is still visible in particle spectra up to 1000 years or so, it is barely noticeable in the emission spectra and morphology, which is in agreement with observations.

We found that the total volumeintegrated particle spectra of the SNR can be very different from single-shock test-particle solutions, especially for very young SNRs. The choice of MF profiles affected the relative contribution to the total spectrum of the RS and the FS, and consequently the emission spectra in the high-energy region varied. Additionally, Alfvénic drift of scattering centers in the shock precursors gives rise to additional spectral feature, whose appearance and time evolution depends on the choice of MF profile. Generally, $\mathrm{P}$ models are more sensitive to Alfvénic drift than D models. Obviously, the models with high MF are affected stronger than those with low MF, for which the effect is subtle.

The particle distributions computed here show a variety of spectral shapes. Interesting is that some of the spectra (both electron and protons) exhibit features which may be considered similiar to ones shown using NDSA, such as spectral concavity and 
high-energy bumps. However, concavity in our spectra arises in a much smaller highenergy band compared to the broadband concavity of NDSA. Besides, it is not reflected in pion-decay spectra as these are produced predominantly by confined particles in the SNR. Additionally, our testparticle spectra are softer at high energies than those of NDSA, even if both are modified by Alfvénic drift, which may provide better agreement with gamma-ray spectra observed from SNRs.

\section{References}

[1] V. L. Ginzburg, S. I. Syrovatsky, Origin of cosmic rays, Progress of Theoretical Physics Supplement 20 (1961) 1-83. doi:10.1143/PTPS.20.1. URL http://ptp.ipap.jp/link?PTPS/20/1/

[2] W. I. Axford, E. Leer, G. Skadron, The acceleration of cosmic rays by shock waves, in: International Cosmic Ray Conference, Vol. 11 of International Cosmic Ray Conference, 1977, pp. 132-137.

[3] G. F. Krymskii, A regular mechanism for the acceleration of charged particles on the front of a shock wave, Akademiia Nauk SSSR Doklady 234 (1977) 1306-1308.

[4] A. R. Bell, The acceleration of cosmic rays in shock fronts. I, MNRAS182 (1978) 147-156.

[5] R. D. Blandford, J. P. Ostriker, Particle acceleration by astrophysical shocks, ApJ221 (1978) L29-L32. doi:10.1086/182658.

[6] P. O. Lagage, C. J. Cesarsky, The maximum energy of cosmic rays accelerated by supernova shocks, A\&A125 (1983) 249-257.

[7] M. A. Malkov, L. O'C Drury, Nonlinear theory of diffusive acceleration of particles by shock waves, Reports on Progress in Physics 64 (2001) 429-481. doi:10.1088/0034-4885/64/4/201.

[8] J. S. Warren, J. P. Hughes, C. Badenes, P. Ghavamian, C. F. McKee, D. Moffett, P. P. Plucinsky, C. Rakowski, E. Reynoso, P. Slane, Cosmic-Ray Acceleration at the Forward Shock in Tycho's Supernova Remnant: Evidence from Chandra X-Ray Observations, ApJ634 (2005) 376389. arXiv:arXiv:astro-ph/0507478, doi:10.1086/496941.

[9] J. C. Raymond, J. Vink, E. A. Helder, A. de Laat, Effects of Neutral Hydrogen on Cosmic Ray Precursors in Supernova Remnant Shock Waves, ArXiv e-printsarXiv:1103.3211.

[10] M. Ave, P. J. Boyle, C. Höppner, J. Marshall, D. Müller, Propagation and Source Energy Spectra of Cosmic Ray Nuclei at High Energies, ApJ697 (2009) 106-114. arXiv:0810.2972, doi:10.1088/0004-637X/697/1/106.

[11] V. S. Ptuskin, F. C. Jones, E. S. Seo, R. Sina, Effect of random nature of cosmic ray sources Supernova remnants on cosmic ray intensity fluctuations, anisotropy, 
and electron energy spectrum, Advances in Space Research 37 (2006) 1909-1912. doi:10.1016/j.asr.2005.08.036.

[12] V. S. Ptuskin, V. N. Zirakashvili, On the spectrum of high-energy cosmic rays produced by supernova remnants in the presence of strong cosmic-ray streaming instability and wave dissipation, A\&A429 (2005) 755-765. arXiv:arXiv:astro-ph/0408025 doi:10.1051/0004-6361:20041517

[13] F. A. Aharonian, A. G. Akhperjanian, K. Aye, A. R. Bazer-Bachi, M. Beilicke, W. Benbow, D. Berge, P. Berghaus, K. Bernlöhr, O. Bolz, C. Boisson, C. Borgmeier, F. Breitling, A. M. Brown, J. Bussons Gordo, P. M. Chadwick, V. R. Chitnis, L. Chounet, R. Cornils, L. Costamante, B. Degrange, A. DjannatiAtaï, L. O. Drury, T. Ergin, P. Espigat, F. Feinstein, P. Fleury, G. Fontaine, S. Funk, Y. A. Gallant, B. Giebels, S. Gillessen, P. Goret, J. Guy, C. Hadjichristidis, M. Hauser, G. Heinzelmann, G. Henri, G. Hermann, J. A. Hinton, W. Hofmann, M. Holleran, D. Horns, O. C. de Jager, I. Jung, B. Khélifi, N. Komin, A. Konopelko, I. J. Latham, R. Le Gallou, M. Lemoine, A. Lemière, N. Leroy, T. Lohse, A. Marcowith, C. Masterson, T. J. L. McComb, M. de Naurois, S. J. Nolan, A. Noutsos, K. J. Orford, J. L. Osborne, M. Ouchrif, M. Panter, G. Pelletier, S. Pita, M. Pohl, G. Pühlhofer, M. Punch, B. C. Raubenheimer, M. Raue, J. Raux, S. M. Rayner, I. Redondo, A. Reimer, O. Reimer, J. Ripken, M. Rivoal, L. Rob, L. Rolland, G. Rowell, V. Sahakian, L. Saugé, S. Schlenker, R. Schlickeiser, C. Schuster, U. Schwanke, M. Siewert, H. Sol, R. Steenkamp, C. Stegmann, J. Tavernet, C. G. Théoret, M. Tluczykont, D. J. van der Walt, G. Vasileiadis, P. Vincent, B. Visser, H. J. Völk, S. J. Wagner, High-energy particle acceleration in the shell of a supernova remnant, Nature432 (2004) 75-77. arXiv:arXiv:astro-ph/0411533 doi:10.1038/nature02960.

[14] F. Aharonian, A. G. Akhperjanian, A. R. Bazer-Bachi, M. Beilicke, W. Benbow, D. Berge, K. Bernlöhr, C. Boisson, O. Bolz, V. Borrel, I. Braun, E. Brion, A. M. Brown, R. Bühler, I. Büsching, S. Carrigan, P. M. Chadwick, L. Chounet, G. Coignet, R. Cornils, L. Costamante, B. Degrange, H. J. Dickinson,
A. Djannati-Ataï, L. O'C. Drury, G. Dubus, K. Egberts, D. Emmanoulopoulos, P. Espigat, F. Feinstein, E. Ferrero, A. Fiasson, G. Fontaine, S. Funk, S. Funk, M. Füßling, Y. A. Gallant, B. Giebels, J. F. Glicenstein, B. Glück, P. Goret, C. Hadjichristidis, D. Hauser, M. Hauser, G. Heinzelmann, G. Henri, G. Hermann, J. A. Hinton, A. Hoffmann, W. Hofmann, M. Holleran, S. Hoppe, D. Horns, A. Jacholkowska, O. C. de Jager, E. Kendziorra, M. Kerschhaggl, B. Khélifi, N. Komin, A. Konopelko, K. Kosack, G. Lamanna, I. J. Latham, R. Le Gallou, A. Lemière, M. Lemoine-Goumard, T. Lohse, J. M. Martin, O. Martineau-Huynh, A. Marcowith, C. Masterson, G. Maurin, T. J. L. McComb, E. Moulin, M. de Naurois, D. Nedbal, S. J. Nolan, A. Noutsos, J. Olive, K. J. Orford, J. L. Osborne, M. Panter, G. Pelletier, S. Pita, G. Pühlhofer, M. Punch, S. Ranchon, B. C. Raubenheimer, M. Raue, S. M. Rayner, A. Reimer, O. Reimer, J. Ripken, L. Rob, L. Rolland, S. Rosier-Lees, G. Rowell, V. Sahakian, A. Santangelo, L. Saugé, S. Schlenker, R. Schlickeiser, R. Schröder, U. Schwanke, S. Schwarzburg, S. Schwemmer, A. Shalchi, H. Sol, D. Spangler, F. Spanier, R. Steenkamp, C. Stegmann, G. Superina, P. H. Tam, J. Tavernet, R. Terrier, M. Tluczykont, C. van Eldik, G. Vasileiadis, C. Venter, J. P. Vialle, P. Vincent, H. J. Völk, S. J. Wagner, M. Ward, Primary particle acceleration above $100 \mathrm{TeV}$ in the shell-type supernova remnant ¡ASTROBJ ¿RX J1713.7-3946i/ASTROBJi, with deep HESS observations, A\&A464 (2007) 235-243. arXiv:arXiv:astro-ph/0611813 doi:10.1051/0004-6361:20066381.

[15] F. Aharonian, A. G. Akhperjanian, A. R. Bazer-Bachi, M. Beilicke, W. Benbow, D. Berge, K. Bernlöhr, C. Boisson, O. Bolz, V. Borrel, I. Braun, A. M. Brown, R. Bühler, I. Büsching, S. Carrigan, P. M. Chadwick, L. Chounet, G. Coignet, R. Cornils, L. Costamante, B. Degrange, H. J. Dickinson, A. Djannati-Ataï, L. O. Drury, G. Dubus, K. Egberts, D. Emmanoulopoulos, P. Espigat, F. Feinstein, E. Ferrero, A. Fiasson, M. D. Filipovic, G. Fontaine, Y. Fukui, S. Funk, S. Funk, M. Füßling, Y. A. Gallant, B. Giebels, J. F. Glicenstein, P. Goret, C. Hadjichristidis, D. Hauser, M. Hauser, 
G. Heinzelmann, G. Henri, G. Hermann, J. A. Hinton, J. S. Hiraga, A. Hoffmann, W. Hofmann, M. Holleran, S. Hoppe, D. Horns, Y. Ishisaki, A. Jacholkowska, O. C. de Jager, E. Kendziorra, M. Kerschhaggl, B. Khélifi, N. Komin, A. Konopelko, K. Kosack, G. Lamanna, I. J. Latham, R. Le Gallou, A. Lemière, M. Lemoine-Goumard, T. Lohse, J. M. Martin, O. Martineau-Huynh, A. Marcowith, C. Masterson, G. Maurin, T. J. L. McComb, E. Moulin, Y. Moriguchi, M. de Naurois, D. Nedbal, S. J. Nolan, A. Noutsos, K. J. Orford, J. L. Osborne, M. Ouchrif, M. Panter, G. Pelletier, S. Pita, G. Pühlhofer, M. Punch, S. Ranchon, B. C. Raubenheimer, M. Raue, S. M. Rayner, A. Reimer, J. Ripken, L. Rob, L. Rolland, S. Rosier-Lees, G. Rowell, V. Sahakian, A. Santangelo, L. Saugé, S. Schlenker, R. Schlickeiser, R. Schröder, U. Schwanke, S. Schwarzburg, S. Schwemmer, A. Shalchi, H. Sol, D. Spangler, F. Spanier, R. Steenkamp, C. Stegmann, G. Superina, P. H. Tam, J. Tavernet, R. Terrier, M. Tluczykont, C. van Eldik, G. Vasileiadis, C. Venter, J. P. Vialle, P. Vincent, H. J. Völk, S. J. Wagner, M. Ward, H.E.S.S. Observations of the Supernova Remnant RX J0852.0-4622: Shell-Type Morphology and Spectrum of a Widely Extended Very High Energy Gamma-Ray Source, ApJ661 (2007) 236-249. arXiv:arXiv:astro-ph/0612495, doi:10.1086/512603.

[16] F. Aharonian, A. G. Akhperjanian, U. B. de Almeida, A. R. Bazer-Bachi, B. Behera, M. Beilicke, W. Benbow, K. Bernlöhr, C. Boisson, A. Bochow, V. Borrel, I. Braun, E. Brion, J. Brucker, R. Bühler, T. Bulik, I. Büsching, T. Boutelier, S. Carrigan, P. M. Chadwick, A. Charbonnier, R. C. G. Chaves, L. Chounet, A. C. Clapson, G. Coignet, L. Costamante, M. Dalton, B. Degrange, H. J. Dickinson, A. Djannati-Ataï, W. Domainko, L. O. Drury, F. Dubois, G. Dubus, J. Dyks, K. Egberts, D. Emmanoulopoulos, P. Espigat, C. Farnier, F. Feinstein, A. Fiasson, A. Förster, G. Fontaine, M. Füßling, S. Gabici, Y. A. Gallant, L. Gérard, B. Giebels, J. F. Glicenstein, B. Glück, P. Goret, C. Hadjichristidis, D. Hauser, M. Hauser, G. Heinzelmann, G. Henri, G. Hermann, J. A. Hinton, A. Hoffmann, W. Hofmann, M. Holleran,
S. Hoppe, D. Horns, A. Jacholkowska, O. C. de Jager, I. Jung, K. Katarzyński, S. Kaufmann, E. Kendziorra, M. Kerschhaggl, D. Khangulyan, B. Khélifi, D. Keogh, N. Komin, K. Kosack, G. Lamanna, I. J. Latham, M. Lemoine-Goumard, J. Lenain, T. Lohse, V. Marandon, J. M. Martin, O. Martineau-Huynh, A. Marcowith, C. Masterson, D. Maurin, T. J. L. McComb, C. Medina, R. Moderski, E. Moulin, M. Naumann-Godo, M. de Naurois, D. Nedbal, D. Nekrassov, J. Niemiec, S. J. Nolan, S. Ohm, J. Olive, E. de Oña Wilhelmi, K. J. Orford, J. L. Osborne, M. Ostrowski, M. Panter, G. Pedaletti, G. Pelletier, P. Petrucci, S. Pita, G. Pühlhofer, M. Punch, A. Quirrenbach, B. C. Raubenheimer, M. Raue, S. M. Rayner, M. Renaud, F. Rieger, J. Ripken, L. Rob, S. Rosier-Lees, G. Rowell, B. Rudak, J. Ruppel, V. Sahakian, A. Santangelo, R. Schlickeiser, F. M. Schöck, R. Schröder, U. Schwanke, S. Schwarzburg, S. Schwemmer, A. Shalchi, J. L. Skilton, H. Sol, D. Spangler, Ł. Stawarz, R. Steenkamp, C. Stegmann, G. Superina, P. H. Tam, J. Tavernet, R. Terrier, O. Tibolla, C. van Eldik, G. Vasileiadis, C. Venter, J. P. Vialle, P. Vincent, J. Vink, M. Vivier, H. J. Völk, F. Volpe, S. J. Wagner, M. Ward, A. A. Zdziarski, A. Zech, Discovery of Gamma-Ray Emission From the Shell-Type Supernova Remnant RCW 86 With Hess, ApJ692 (2009) 1500-1505. arXiv:0810.2689, doi:10.1088/0004-637X/692/2/1500

[17] F. Acero, F. Aharonian, A. G. Akhperjanian, G. Anton, U. Barres de Almeida, A. R. Bazer-Bachi, Y. Becherini, B. Behera, M. Beilicke, K. Bernlöhr, A. Bochow, C. Boisson, J. Bolmont, V. Borrel, J. Brucker, F. Brun, P. Brun, R. Bühler, T. Bulik, I. Büsching, T. Boutelier, P. M. Chadwick, A. Charbonnier, R. C. G. Chaves, A. Cheesebrough, J. Conrad, L. Chounet, A. C. Clapson, G. Coignet, M. Dalton, M. K. Daniel, I. D. Davids, B. Degrange, C. Deil, H. J. Dickinson, A. DjannatiAtaï, W. Domainko, L. O'C. Drury, F. Dubois, G. Dubus, J. Dyks, M. Dyrda, K. Egberts, P. Eger, P. Espigat, L. Fallon, C. Farnier, S. Fegan, F. Feinstein, A. Fiasson, A. Förster, G. Fontaine, M. Füßling, S. Gabici, Y. A. Gallant, L. Gérard, D. Gerbig, B. Giebels, J. F. Glicenstein, B. Glück, P. Goret, D. Göring, 
D. Hauser, M. Hauser, S. Heinz, G. Heinzelmann, G. Henri, G. Hermann, J. A. Hinton, A. Hoffmann, W. Hofmann, P. Hofverberg, M. Holleran, S. Hoppe, D. Horns, A. Jacholkowska, O. C. de Jager, C. Jahn, I. Jung, K. Katarzyński, U. Katz, S. Kaufmann, M. Kerschhaggl, D. Khangulyan, B. Khélifi, D. Keogh, D. Klochkov, W. Kluźniak, T. Kneiske, N. Komin, K. Kosack, R. Kossakowski, G. Lamanna, M. Lemoine-Goumard, J. Lenain, T. Lohse, V. Marandon, A. Marcowith, J. Masbou, D. Maurin, T. J. L. McComb, M. C. Medina, J. Méhault, R. Moderski, E. Moulin, M. Naumann-Godo, M. de Naurois, D. Nedbal, D. Nekrassov, B. Nicholas, J. Niemiec, S. J. Nolan, S. Ohm, J. Olive, E. de Oña Wilhelmi, K. J. Orford, M. Ostrowski, M. Panter, M. Paz Arribas, G. Pedaletti, G. Pelletier, P. Petrucci, S. Pita, G. Pühlhofer, M. Punch, A. Quirrenbach, B. C. Raubenheimer, M. Raue, S. M. Rayner, O. Reimer, M. Renaud, R. de Los Reyes, F. Rieger, J. Ripken, L. Rob, S. Rosier-Lees, G. Rowell, B. Rudak, C. B. Rulten, J. Ruppel, F. Ryde, V. Sahakian, A. Santangelo, R. Schlickeiser, F. M. Schöck, A. Schönwald, U. Schwanke, S. Schwarzburg, S. Schwemmer, A. Shalchi, I. Sushch, M. Sikora, J. L. Skilton, H. Sol, E. Stawarz, R. Steenkamp, C. Stegmann, F. Stinzing, G. Superina, A. Szostek, P. H. Tam, J. Tavernet, R. Terrier, O. Tibolla, M. Tluczykont, C. van Eldik, G. Vasileiadis, C. Venter, L. Venter, J. P. Vialle, P. Vincent, J. Vink, M. Vivier, H. J. Völk, F. Volpe, S. Vorobiov, S. J. Wagner, M. Ward, A. A. Zdziarski, A. Zech, HESS Collaboration, First detection of VHE $\gamma$-rays from SN 1006 by HESS, A\&A516 (2010) A62+. arXiv:1004.2124, doi:10.1051/0004-6361/200913916.

[18] V. A. Acciari, E. Aliu, T. Arlen, T. Aune, M. Bautista, M. Beilicke, W. Benbow, D. Boltuch, S. M. Bradbury, J. H. Buckley, V. Bugaev, Y. Butt, K. Byrum, A. Cannon, A. Cesarini, Y. C. Chow, L. Ciupik, P. Cogan, W. Cui, R. Dickherber, C. Duke, T. Ergin, S. J. Fegan, J. P. Finley, G. Finnegan, P. Fortin, L. Fortson, A. Furniss, N. Galante, D. Gall, G. H. Gillanders, J. Grube, R. Guenette, G. Gyuk, D. Hanna, J. Holder, D. Huang, C. M. Hui, T. B. Hu- mensky, P. Kaaret, N. Karlsson, M. Kertzman, D. Kieda, A. Konopelko, H. Krawczynski, F. Krennrich, M. J. Lang, S. LeBohec, G. Maier, S. McArthur, A. McCann, M. McCutcheon, J. Millis, P. Moriarty, R. A. Ong, D. Pandel, J. S. Perkins, M. Pohl, J. Quinn, K. Ragan, P. T. Reynolds, E. Roache, H. J. Rose, M. Schroedter, G. H. Sembroski, A. W. Smith, B. R. Smith, D. Steele, S. P. Swordy, M. Theiling, S. Thibadeau, A. Varlotta, V. V. Vassiliev, S. Vincent, R. G. Wagner, S. P. Wakely, J. E. Ward, T. C. Weekes, A. Weinstein, T. Weisgarber, S. Wissel, M. Wood, VERITAS Collaboration, Observations of the Shell-type Supernova Remnant Cassiopeia A at TeV Energies with VERITAS, ApJ714 (2010) 163-169. arXiv:1002.2974, doi:10.1088/0004-637X/714/1/163.

[19] A. A. Abdo, M. Ackermann, M. Ajello, A. Allafort, L. Baldini, J. Ballet, G. Barbiellini, M. G. Baring, D. Bastieri, B. M. Baughman, K. Bechtol, R. Bellazzini, B. Berenji, R. D. Blandford, E. D. Bloom, E. Bonamente, A. W. Borgland, J. Bregeon, A. Brez, M. Brigida, P. Bruel, R. Buehler, T. H. Burnett, G. Busetto, G. A. Caliandro, R. A. Cameron, P. A. Caraveo, J. M. Casandjian, C. Cecchi, Ö. Çelik, E. Charles, S. Chaty, A. Chekhtman, C. C. Cheung, J. Chiang, A. N. Cillis, S. Ciprini, R. Claus, J. Cohen-Tanugi, J. Conrad, S. Corbel, F. de Palma, S. W. Digel, M. Dormody, E. d. C. e. Silva, P. S. Drell, R. Dubois, D. Dumora, Y. Edmonds, C. Farnier, C. Favuzzi, S. J. Fegan, E. C. Ferrara, W. B. Focke, P. Fortin, M. Frailis, Y. Fukazawa, S. Funk, P. Fusco, F. Gargano, D. Gasparrini, N. Gehrels, S. Germani, G. Giavitto, N. Giglietto, F. Giordano, T. Glanzman, G. Godfrey, I. A. Grenier, M. Grondin, J. E. Grove, L. Guillemot, S. Guiriec, Y. Hanabata, E. Hays, A. K. Harding, M. Hayashida, D. Horan, R. E. Hughes, M. S. Jackson, A. S. Johnson, T. J. Johnson, W. N. Johnson, T. Kamae, H. Katagiri, J. Kataoka, N. Kawai, M. Kerr, J. Knödlseder, M. Kuss, J. Lande, L. Latronico, M. Lemoine-Goumard, F. Longo, F. Loparco, B. Lott, M. N. Lovellette, P. Lubrano, A. Makeev, M. N. Mazziotta, C. Meurer, P. F. Michelson, W. Mitthumsiri, T. Mizuno, C. Monte, M. E. Monzani, A. Morselli, I. V. Moskalenko, S. Mur- 
gia, T. Nakamori, P. L. Nolan, J. P. Norris, E. Nuss, T. Ohsugi, A. Okumura, N. Omodei, E. Orlando, J. F. Ormes, D. Paneque, J. H. Panetta, V. Pelassa, M. Pepe, M. PesceRollins, F. Piron, M. Pohl, T. A. Porter, S. Rainò, R. Rando, A. Reimer, O. Reimer, T. Reposeur, S. Ritz, A. Y. Rodriguez, R. W. Romani, M. Roth, H. Sadrozinski, A. Sander, P. M. Saz Parkinson, J. D. Scargle, C. Sgrò, E. J. Siskind, D. A. Smith, P. D. Smith, P. Spinelli, M. S. Strickman, D. J. Suson, H. Tajima, T. Takahashi, T. Tanaka, J. B. Thayer, J. G. Thayer, D. J. Thompson, S. E. Thorsett, L. Tibaldo, O. Tibolla, D. F. Torres, G. Tosti, A. Tramacere, Y. Uchiyama, T. L. Usher, A. Van Etten, V. Vasileiou, C. Venter, N. Vilchez, V. Vitale, A. P. Waite, P. Wang, B. L. Winer, K. S. Wood, R. Yamazaki, T. Ylinen, M. Ziegler, Fermi-Lat Discovery of GeV Gamma-Ray Emission from the Young Supernova Remnant Cassiopeia A, ApJ710 (2010) L92-L97. arXiv:1001.1419. doi:10.1088/2041-8205/710/1/L92.

[20] V. A. Acciari, E. Aliu, T. Arlen, T. Aune, M. Bautista, M. Beilicke, W. Benbow, S. M. Bradbury, J. H. Buckley, V. Bugaev, Y. Butt, K. Byrum, A. Cannon, O. Celik, A. Cesarini, Y. C. Chow, L. Ciupik, P. Cogan, P. Colin, W. Cui, M. K. Daniel, R. Dickherber, C. Duke, V. V. Dwarkadas, T. Ergin, S. J. Fegan, J. P. Finley, G. Finnegan, P. Fortin, L. Fortson, A. Furniss, D. Gall, K. Gibbs, G. H. Gillanders, S. Godambe, J. Grube, R. Guenette, G. Gyuk, D. Hanna, E. Hays, J. Holder, D. Horan, C. M. Hui, T. B. Humensky, A. Imran, P. Kaaret, N. Karlsson, M. Kertzman, D. Kieda, J. Kildea, A. Konopelko, H. Krawczynski, F. Krennrich, M. J. Lang, S. LeBohec, G. Maier, A. McCann, M. McCutcheon, J. Millis, P. Moriarty, R. A. Ong, A. N. Otte, D. Pandel, J. S. Perkins, M. Pohl, J. Quinn, K. Ragan, L. C. Reyes, P. T. Reynolds, E. Roache, H. J. Rose, M. Schroedter, G. H. Sembroski, A. W. Smith, D. Steele, S. P. Swordy, M. Theiling, J. A. Toner, L. Valcarcel, A. Varlotta, V. V. Vassiliev, S. Vincent, R. G. Wagner, S. P. Wakely, J. E. Ward, T. C. Weekes, A. Weinstein, T. Weisgarber, D. A. Williams, S. Wissel, M. Wood, B. Zitzer, Observation of Extended Very High Energy Emission from the
Supernova Remnant IC 443 with VERITAS, ApJ698 (2009) L133-L137. arXiv:0905.3291, doi:10.1088/0004-637X/698/2/L133

[21] A. A. Abdo, M. Ackermann, M. Ajello, L. Baldini, J. Ballet, G. Barbiellini, D. Bastieri, B. M. Baughman, K. Bechtol, R. Bellazzini, B. Berenji, R. D. Blandford, E. D. Bloom, E. Bonamente, A. W. Borgland, J. Bregeon, A. Brez, M. Brigida, P. Bruel, T. H. Burnett, S. Buson, G. A. Caliandro, R. A. Cameron, P. A. Caraveo, J. M. Casandjian, C. Cecchi, Ö. Çelik, A. Chekhtman, C. C. Cheung, J. Chiang, A. N. Cillis, S. Ciprini, R. Claus, J. Cohen-Tanugi, L. R. Cominsky, J. Conrad, S. Cutini, C. D. Dermer, A. de Angelis, F. de Palma, E. d. C. e. Silva, P. S. Drell, A. DrlicaWagner, R. Dubois, D. Dumora, C. Farnier, C. Favuzzi, S. J. Fegan, W. B. Focke, P. Fortin, M. Frailis, Y. Fukazawa, S. Funk, P. Fusco, F. Gargano, D. Gasparrini, N. Gehrels, S. Germani, G. Giavitto, B. Giebels, N. Giglietto, F. Giordano, T. Glanzman, G. Godfrey, I. A. Grenier, M. Grondin, J. E. Grove, L. Guillemot, S. Guiriec, Y. Hanabata, A. K. Harding, M. Hayashida, R. E. Hughes, M. S. Jackson, G. Jóhannesson, A. S. Johnson, T. J. Johnson, W. N. Johnson, T. Kamae, H. Katagiri, J. Kataoka, N. Kawai, M. Kerr, J. Knödlseder, M. L. Kocian, M. Kuss, J. Lande, L. Latronico, S. Lee, M. LemoineGoumard, F. Longo, F. Loparco, B. Lott, M. N. Lovellette, P. Lubrano, G. M. Madejski, A. Makeev, M. N. Mazziotta, C. Meurer, P. F. Michelson, W. Mitthumsiri, A. A. Moiseev, C. Monte, M. E. Monzani, A. Morselli, I. V. Moskalenko, S. Murgia, T. Nakamori, P. L. Nolan, J. P. Norris, E. Nuss, T. Ohsugi, E. Orlando, J. F. Ormes, M. Ozaki, D. Paneque, J. H. Panetta, D. Parent, V. Pelassa, M. Pepe, M. Pesce-Rollins, F. Piron, T. A. Porter, S. Rainò, R. Rando, M. Razzano, A. Reimer, O. Reimer, T. Reposeur, L. S. Rochester, A. Y. Rodriguez, R. W. Romani, M. Roth, F. Ryde, H. Sadrozinski, D. Sanchez, A. Sander, P. M. Saz Parkinson, J. D. Scargle, C. Sgrò, E. J. Siskind, D. A. Smith, P. D. Smith, G. Spandre, P. Spinelli, M. S. Strickman, A. W. Strong, D. J. Suson, H. Tajima, H. Takahashi, T. Takahashi, T. Tanaka, J. B. Thayer, J. G. Thayer, D. J. Thompson, L. Tibaldo, D. F. Torres, G. Tosti, A. Tra- 
macere, Y. Uchiyama, T. L. Usher, A. Van Etten, V. Vasileiou, C. Venter, N. Vilchez, V. Vitale, A. P. Waite, P. Wang, B. L. Winer, K. S. Wood, T. Ylinen, M. Ziegler, Observation of Supernova Remnant IC 443 with the Fermi Large Area Telescope, ApJ712 (2010) 459-468. arXiv:1002.2198, doi:10.1088/0004-637X/712/1/459.

[22] D. Castro, P. Slane, Fermi Large Area Telescope Observations of Supernova Remnants Interacting with Molecular Clouds, ApJ717 (2010) 372-378. arXiv:1002.2738, doi:10.1088/0004-637X/717/1/372.

[23] A. A. Abdo, M. Ackermann, M. Ajello, L. Baldini, J. Ballet, G. Barbiellini, M. G. Baring, D. Bastieri, B. M. Baughman, K. Bechtol, R. Bellazzini, B. Berenji, R. D. Blandford, E. D. Bloom, E. Bonamente, A. W. Borgland, J. Bregeon, A. Brez, M. Brigida, P. Bruel, T. H. Burnett, S. Buson, G. A. Caliandro, R. A. Cameron, P. A. Caraveo, J. M. Casandjian, C. Cecchi, Ö. Çelik, A. Chekhtman, C. C. Cheung, J. Chiang, S. Ciprini, R. Claus, I. Cognard, J. Cohen-Tanugi, L. R. Cominsky, J. Conrad, S. Cutini, C. D. Dermer, A. de Angelis, F. de Palma, S. W. Digel, E. do Couto e Silva, P. S. Drell, R. Dubois, D. Dumora, C. Espinoza, C. Farnier, C. Favuzzi, S. J. Fegan, W. B. Focke, P. Fortin, M. Frailis, Y. Fukazawa, S. Funk, P. Fusco, F. Gargano, D. Gasparrini, N. Gehrels, S. Germani, G. Giavitto, B. Giebels, N. Giglietto, F. Giordano, T. Glanzman, G. Godfrey, I. A. Grenier, M. Grondin, J. E. Grove, L. Guillemot, S. Guiriec, Y. Hanabata, A. K. Harding, M. Hayashida, E. Hays, R. E. Hughes, M. S. Jackson, G. Jóhannesson, A. S. Johnson, T. J. Johnson, W. N. Johnson, T. Kamae, H. Katagiri, J. Kataoka, J. Katsuta, N. Kawai, M. Kerr, J. Knödlseder, M. L. Kocian, M. Kramer, M. Kuss, J. Lande, L. Latronico, M. Lemoine-Goumard, F. Longo, F. Loparco, B. Lott, M. N. Lovellette, P. Lubrano, A. G. Lyne, G. M. Madejski, A. Makeev, M. N. Mazziotta, J. E. McEnery, C. Meurer, P. F. Michelson, W. Mitthumsiri, T. Mizuno, C. Monte, M. E. Monzani, A. Morselli, I. V. Moskalenko, S. Murgia, T. Nakamori, P. L. Nolan, J. P. Norris, A. Noutsos, E. Nuss, T. Ohsugi, N. Omodei, E. Orlando, J. F. Ormes, D. Paneque, D. Parent, V. Pelassa,
M. Pepe, M. Pesce-Rollins, F. Piron, T. A. Porter, S. Rainò, R. Rando, M. Razzano, A. Reimer, O. Reimer, T. Reposeur, L. S. Rochester, A. Y. Rodriguez, R. W. Romani, M. Roth, F. Ryde, H. Sadrozinski, D. Sanchez, A. Sander, P. M. S. Parkinson, J. D. Scargle, C. Sgrò, E. J. Siskind, D. A. Smith, P. D. Smith, G. Spandre, P. Spinelli, B. W. Stappers, F. W. Stecker, M. S. Strickman, D. J. Suson, H. Tajima, H. Takahashi, T. Takahashi, T. Tanaka, J. B. Thayer, J. G. Thayer, G. Theureau, D. J. Thompson, L. Tibaldo, O. Tibolla, D. F. Torres, G. Tosti, A. Tramacere, Y. Uchiyama, T. L. Usher, V. Vasileiou, C. Venter, N. Vilchez, V. Vitale, A. P. Waite, P. Wang, B. L. Winer, K. S. Wood, R. Yamazaki, T. Ylinen, M. Ziegler, Gamma-Ray Emission from the Shell of Supernova Remnant W44 Revealed by the Fermi LAT, Science 327 (2010) 1103-. doi:10.1126/science.1182787

[24] A. A. Abdo, M. Ackermann, M. Ajello, A. Allafort, L. Baldini, J. Ballet, G. Barbiellini, D. Bastieri, K. Bechtol, R. Bellazzini, B. Berenji, R. D. Blandford, E. D. Bloom, E. Bonamente, A. W. Borgland, A. Bouvier, T. J. Brandt, J. Bregeon, M. Brigida, P. Bruel, R. Buehler, S. Buson, G. A. Caliandro, R. A. Cameron, P. A. Caraveo, S. Carrigan, J. M. Casandjian, C. Cecchi, Ö. Çelik, A. Chekhtman, J. Chiang, S. Ciprini, R. Claus, J. CohenTanugi, J. Conrad, C. D. Dermer, F. de Palma, E. d. C. e. Silva, P. S. Drell, R. Dubois, D. Dumora, C. Farnier, C. Favuzzi, S. J. Fegan, Y. Fukazawa, Y. Fukui, S. Funk, P. Fusco, F. Gargano, N. Gehrels, S. Germani, N. Giglietto, F. Giordano, T. Glanzman, G. Godfrey, I. A. Grenier, J. E. Grove, S. Guiriec, D. Hadasch, Y. Hanabata, A. K. Harding, E. Hays, D. Horan, R. E. Hughes, G. Jóhannesson, A. S. Johnson, W. N. Johnson, T. Kamae, H. Katagiri, J. Kataoka, J. Knödlseder, M. Kuss, J. Lande, L. Latronico, S. Lee, M. Lemoine-Goumard, M. Llena Garde, F. Longo, F. Loparco, M. N. Lovellette, P. Lubrano, A. Makeev, M. N. Mazziotta, P. F. Michelson, W. Mitthumsiri, T. Mizuno, A. A. Moiseev, C. Monte, M. E. Monzani, A. Morselli, I. V. Moskalenko, S. Murgia, T. Nakamori, P. L. Nolan, J. P. Norris, E. Nuss, M. Ohno, T. Ohsugi, N. Omodei, 
E. Orlando, J. F. Ormes, M. Ozaki, J. H. Panetta, D. Parent, V. Pelassa, M. Pepe, M. Pesce-Rollins, F. Piron, T. A. Porter, S. Rainò, R. Rando, M. Razzano, A. Reimer, O. Reimer, T. Reposeur, A. Y. Rodriguez, M. Roth, H. Sadrozinski, A. Sander, P. M. Saz Parkinson, C. Sgrò, E. J. Siskind, D. A. Smith, P. D. Smith, G. Spandre, P. Spinelli, M. S. Strickman, D. J. Suson, H. Tajima, H. Takahashi, T. Takahashi, T. Tanaka, J. B. Thayer, J. G. Thayer, D. J. Thompson, L. Tibaldo, O. Tibolla, D. F. Torres, G. Tosti, Y. Uchiyama, T. Uehara, T. L. Usher, V. Vasileiou, N. Vilchez, V. Vitale, A. P. Waite, P. Wang, B. L. Winer, K. S. Wood, H. Yamamoto, R. Yamazaki, Z. Yang, T. Ylinen, M. Ziegler, Fermi Large Area Telescope Observations of the Supernova Remnant W28 (G6.4-0.1), ApJ718 (2010) 348-356. doi : 10.1088/0004-637X/718/1/348.

[25] A. R. Bell, Turbulent amplification of magnetic field and diffusive shock acceleration of cosmic rays, MNRAS353 (2004) 550-558. doi:10.1111/j.1365-2966.2004.08097.x.

[26] V. N. Zirakashvili, V. S. Ptuskin, Diffusive Shock Acceleration with Magnetic Amplification by Nonresonant Streaming Instability in Supernova Remnants, ApJ678 (2008) 939-949. arXiv:0801.4488, doi:10.1086/529580.

[27] E. Amato, P. Blasi, A kinetic approach to cosmic-ray-induced streaming instability at supernova shocks, MNRAS392 (2009) 1591-1600. arXiv:0806.1223, doi:10.1111/j.1365-2966.2008.14200.x.

[28] R. A. Chevalier, Magnetic field amplification in interstellar collisionless shock waves, Nature266 (1977) 701-+. doi:10.1038/266701a0

[29] J. Giacalone, J. R. Jokipii, Magnetic Field Amplification by Shocks in Turbulent Fluids, ApJ663 (2007) L41-L44. doi:10.1086/519994.

[30] A. Beresnyak, T. W. Jones, A. Lazarian, Turbulence-Induced Magnetic Fields and Structure of Cosmic Ray Modified Shocks, ApJ707 (2009) 1541-1549. arXiv:0908.2806, doi:10.1088/0004-637X/707/2/1541.

[31] J. Skilling, Cosmic ray streaming. I - Effect of Alfven waves on particles, MNRAS172 (1975) 557-566.

[32] V. N. Zirakashvili, V. S. Ptuskin, The influ- ence of the Alfvénic drift on the shape of cosmic ray spectra in SNRs, in: F. A. Aharonian, W. Hofmann, \& F. Rieger (Ed.), American Institute of Physics Conference Series, Vol. 1085 of American Institute of Physics Conference Series, 2008, pp. 336-339. arXiv:0807.2754, doi:10.1063/1.3076675

[33] V. Ptuskin, V. Zirakashvili, E. Seo, Spectrum of Galactic Cosmic Rays Accelerated in Supernova Remnants, ApJ718 (2010) 31-36. arXiv:1006.0034 doi:10.1088/0004-637X/718/1/31.

[34] H. Kang, D. Ryu, Diffusive Shock Acceleration in Test-particle Regime, ApJ721 (2010) 886-892. arXiv:1008.0429 doi:10.1088/0004-637X/721/1/886

[35] D. Caprioli, P. Blasi, E. Amato, M. Vietri, Dynamical feedback of self-generated magnetic fields in cosmic ray modified shocks, MNRAS395 (2009) 895-906. arXiv:0807.4261, doi:10.1111/j.1365-2966.2009.14570.x

[36] D. Caprioli, P. Blasi, E. Amato, Nonlinear diffusive acceleration of heavy nuclei in supernova remnant shocks, Astroparticle Physics 34 (2011) 447-456. arXiv:1007.1925, doi:10.1016/j.astropartphys.2010.10.011.

[37] S. P. Reynolds, Supernova Remnants at High Energy, ARA\&A46 (2008) 89-126. doi:10.1146/annurev.astro.46.060407.145237.

[38] E. V. Gotthelf, B. Koralesky, L. Rudnick, T. W. Jones, U. Hwang, R. Petre, Chandra Detection of the Forward and Reverse Shocks in Cassiopeia A, ApJ552 (2001) L39-L43. arXiv:arXiv:astro-ph/0104161, doi:10.1086/320250.

[39] E. A. Helder, J. Vink, Characterizing the Nonthermal Emission of Cassiopeia A, ApJ686 (2008) 1094-1102. arXiv:0806.3748, doi:10.1086/591242.

[40] M. Sasaki, T. J. Gaetz, W. P. Blair, R. J. Edgar, J. A. Morse, P. P. Plucinsky, R. K. Smith, Far-Ultraviolet and X-Ray Observations of the Reverse Shock in the Small Magellanic Cloud Supernova Remnant 1E 0102.2-7219, ApJ642 (2006) 260-269. arXiv:arXiv:astro-ph/0602094, doi:10.1086/500789.

[41] J. Rho, K. K. Dyer, K. J. Borkowski, S. P. Reynolds, X-Ray Synchrotronemitting Fe-rich Ejecta in Supernova Remnant RCW 86, ApJ581 (2002) 1116- 
1131. arXiv:arXiv:astro-ph/0208013 doi:10.1086/344248.

[42] T. DeLaney, B. Koralesky, L. Rudnick, J. R. Dickel, Radio Spectral Index Variations and Physical Conditions in Kepler's Supernova Remnant, ApJ580 (2002) 914-927. arXiv:arXiv:astro-ph/0210355 doi:10.1086/343787.

[43] D. C. Ellison, A. Decourchelle, J. Ballet, Nonlinear particle acceleration at reverse shocks in supernova remnants, A\&A429 (2005) 569-580. arXiv:arXiv:astro-ph/0409182 doi:10.1051/0004-6361:20041668

[44] V. N. Zirakashvili, F. A. Aharonian, Nonthermal Radiation of Young Supernova Remnants: The Case of RX J1713.7-3946, ApJ708 (2010) 965-980. arXiv:0909.2285, doi:10.1088/0004-637X/708/2/965.

[45] K. M. Schure, A. Achterberg, R. Keppens, J. Vink, Time-dependent particle acceleration in supernova remnants in different environments, MNRAS406 (2010) 2633-2649. arXiv:1004.2766, doi:10.1111/j.1365-2966.2010.16857.x

[46] V. A. Acciari, E. Aliu, T. Arlen, T. Aune, M. Beilicke, W. Benbow, S. M. Bradbury, J. H. Buckley, V. Bugaev, K. Byrum, A. Cannon, A. Cesarini, L. Ciupik, E. Collins-Hughes, W. Cui, R. Dickherber, C. Duke, M. Errando, J. P. Finley, G. Finnegan, L. Fortson, A. Furniss, N. Galante, D. Gall, G. H. Gillanders, S. Godambe, S. Griffin, J. Grube, R. Guenette, G. Gyuk, D. Hanna, J. Holder, J. P. Hughes, C. M. Hui, T. B. Humensky, P. Kaaret, N. Karlsson, M. Kertzman, D. Kieda, H. Krawczynski, F. Krennrich, M. J. Lang, S. LeBohec, A. S. Madhavan, G. Maier, P. Majumdar, S. McArthur, A. McCann, P. Moriarty, R. Mukherjee, R. A. Ong, M. Orr, A. N. Otte, D. Pandel, N. H. Park, J. S. Perkins, M. Pohl, J. Quinn, K. Ragan, L. C. Reyes, P. T. Reynolds, E. Roache, H. J. Rose, D. B. Saxon, M. Schroedter, G. H. Sembroski, G. D. Senturk, P. Slane, A. W. Smith, G. Tešić, M. Theiling, S. Thibadeau, K. Tsurusaki, A. Varlotta, V. V. Vassiliev, S. Vincent, M. Vivier, S. P. Wakely, J. E. Ward, T. C. Weekes, A. Weinstein, T. Weisgarber, D. A. Williams, M. Wood, B. Zitzer, Discovery of $\mathrm{TeV}$ Gamma-ray Emission from Tycho's Supernova Remnant,
ApJ730 (2011) L20+. arXiv:1102.3871, doi:10.1088/2041-8205/730/2/L20.

[47] H. J. Völk, E. G. Berezhko, L. T. Ksenofontov, Magnetic field amplification in Tycho and other shell-type supernova remnants, A\&A433 (2005) 229-240. doi:10.1051/0004-6361:20042015.

[48] A. R. Bell, S. G. Lucek, Cosmic ray acceleration to very high energy through the nonlinear amplification by cosmic rays of the seed magnetic field, MNRAS321 (2001) 433-438. doi:10.1046/j.1365-8711.2001.04063.x.

[49] E. G. Berezhko, D. C. Ellison, A Simple Model of Nonlinear Diffusive Shock Acceleration, ApJ526 (1999) 385-399. doi:10.1086/307993.

[50] P. Blasi, S. Gabici, G. Vannoni, On the role of injection in kinetic approaches to non-linear particle acceleration at nonrelativistic shock waves, MNRAS361 (2005) 907-918. arXiv:arXiv:astro-ph/0505351, doi:10.1111/j.1365-2966.2005.09227.x.

[51] B. Reville, J. G. Kirk, P. Duffy, Steady-State Solutions in Nonlinear Diffusive Shock Acceleration, ApJ694 (2009) 951-958. arXiv:0812.3993, doi:10.1088/0004-637X/694/2/951

[52] E. G. Berezhko, V. K. Yelshin, L. T. Ksenofontov, Numerical investigation of cosmic ray acceleration in supernova remnants, Astroparticle Physics 2 (1994) 215-227. doi:10.1016/0927-6505(94)90043-4

[53] H. Kang, T. W. Jones, Numerical studies of diffusive shock acceleration at spherical shocks, Astroparticle Physics 25 (2006) 246-258. arXiv:arXiv:astro-ph/0603223, doi:10.1016/j.astropartphys.2006.02.006.

[54] J. E. Guyer, D. Wheeler, J. A. Warren, Fipy: Partial differential equations with python, Computing in Science and Engineering 11 (2009) 6-15. doi:http://doi.ieeecomputersociety.org/10.1109/MCS

[55] J. M. Blondin, P. Lundqvist, Formation of the circumstellar shell around SN 1987A, ApJ405 (1993) 337-352. doi:10.1086/172366.

[56] P. Colella, P. R. Woodward, The Piecewise Parabolic Method (PPM) for GasDynamical Simulations, Journal of Computational Physics 54 (1984) 174-201. doi:10.1016/0021-9991(84)90143-8.

[57] V. V. Dwarkadas, The Evolution of Su- 
pernovae in Circumstellar Wind-Blown Bubbles. I. Introduction and OneDimensional Calculations, ApJ630 (2005) 892-910. arXiv:arXiv:astro-ph/0410464 doi:10.1086/432109.

[58] V. V. Dwarkadas, The Evolution of Supernovae in Circumstellar Wind Bubbles. II. Case of a Wolf-Rayet Star, ApJ667 (2007) 226-247. arXiv:0706.1049, doi:10.1086/520670.

[59] V. V. Dwarkadas, R. A. Chevalier, Interaction of Type IA Supernovae with Their Surroundings, ApJ497 (1998) 807-+. doi:10.1086/305478.

[60] A. Vladimirov, D. C. Ellison, A. Bykov, Nonlinear Diffusive Shock Acceleration with Magnetic Field Amplification, ApJ652 (2006) 1246-1258. arXiv: arXiv:astro-ph/0606433, doi:10.1086/508154.

[61] A. M. Bykov, D. C. Ellison, M. Renaud, Magnetic Fields in Cosmic Particle Acceleration Sources, Space Sci. Rev.(2011) 32-+arXiv:1105.0130, doi:10.1007/s11214-011-9761-4.

[62] H. J. Völk, E. G. Berezhko, L. T. Ksenofontov, Internal dynamics and particle acceleration in Tycho's SNR, A\&A483 (2008) 529-535. arXiv:0803.1403, doi:10.1051/0004-6361:20079337

[63] R. A. Chevalier, The radio and X-ray emission from type II supernovae, ApJ259 (1982) 302310. doi:10.1086/160167.

[64] K. W. Weiler, N. Panagia, M. J. Montes, R. A. Sramek, Radio Emission from Supernovae and Gamma-Ray Bursters, ARA\&A40 (2002) 387-438. doi:10.1146/annurev.astro.40.060401.093744

[65] I. Lerche, M. Pohl, R. Schlickeiser, Turbulent adiabatic shock waves and diffusive particle acceleration, Journal of Plasma Physics 64 (2000) 459-474.

[66] M. A. Riquelme, A. Spitkovsky, Nonlinear Study of Bell's Cosmic Ray Current-Driven Instability, ApJ694 (2009) 626-642. arXiv:0810.4565, doi:10.1088/0004-637X/694/1/626.

[67] T. Stroman, M. Pohl, J. Niemiec, Kinetic Simulations of Turbulent MagneticField Growth by Streaming Cosmic Rays, ApJ706 (2009) 38-44. arXiv:0909.5212, doi:10.1088/0004-637X/706/1/38
[68] M. A. Riquelme, A. Spitkovsky, Magnetic Amplification by Magnetized Cosmic Rays in Supernova Remnant Shocks, ApJ717 (2010) 1054-1066. arXiv:0912.4990, doi:10.1088/0004-637X/717/2/1054

[69] L. O. Drury, An introduction to the theory of diffusive shock acceleration of energetic particles in tenuous plasmas, Reports on Progress in Physics 46 (1983) 973-1027. doi:10.1088/0034-4885/46/8/002.

[70] H. Kang, Energy Spectrum Of Nonthermal Electrons Accelerated At A Plane Shock, ArXiv e-printsarXiv:1102.3109.

[71] S. J. Sturner, J. G. Skibo, C. D. Dermer, J. R. Mattox, Temporal Evolution of Nonthermal Spectra from Supernova Remnants, ApJ490 (1997) 619-+. doi:10.1086/304894

[72] M. Pohl, Leptonic origin of TeV gamma-rays from supernova remnants., A\&A307 (1996) L57-L59. arXiv:arXiv:astro-ph/9602115.

[73] F. A. Aharonian, Very high energy cosmic gamma radiation : a crucial window on the extreme Universe, 2004.

[74] C. Huang, S. Park, M. Pohl, C. D. Daniels, Gamma-rays produced in cosmic-ray interactions and the TeV-band spectrum of RX J1713.7-3946, Astroparticle Physics 27 (2007) 429-439. arXiv:arXiv:astro-ph/0611854 doi:10.1016/j.astropartphys.2007.01.002. 